\title{
A Geometric Approach to Fault Detection and Isolation of Continuous-time Markovian Jump Linear Systems
}

\author{
N. Meskin, Student Member, IEEE and K. Khorasani, Member, IEEE
}

\begin{abstract}
This paper is concerned with development of novel fault detection and isolation (FDI) strategies for the Markovian jump linear systems (MJLS's) and the MJLS's with timedelays (MJLSD's). First a geometric property that is related to the unobservable subspace of MJLS's is presented. The notion of a finite unobservable subspace is then introduced for the MJLSD's. The concept of unobservability subspace is introduced for both the MJLS's and the MJLSD's and an algorithm for its construction is described. The necessary and sufficient conditions for solvability of the fundamental problem of residual generation (FPRG) for the MJLS's are developed by utilizing our introduced unobservability subspace. Furthermore, sufficient solvability conditions of the FPRG for the MJLSD's are also derived. Finally, sufficient conditions for designing an $H_{\infty}$ based FDI algorithm for the MJLS's with an unknown transition matrix that are also subject to input and output disturbances are developed.
\end{abstract}

Keywords: Fault detection and isolation (FDI), Markovian jump systems, Unobservability subspaces, Geometric FDI, Time-delay systems.

\section{INTRODUCTION}

In the past three decades various model-based approaches for fault detection and isolation (FDI) of linear systems have been developed in the literature [1]-[4]. One way of enhancing the performance of FDI algorithms involves generating residuals that in response to a particular fault only a fault-specific subset of residuals becomes nonzero. This is designated as the structured residual set [5]. In this paper, we develop a geometric framework for design of structured residual set for Markovian jump linear systems (MJLS's) and MJLS's with time-delays (MJLSD's).

A great deal of attention has recently been devoted to MJLS's [6]-[9] which comprise an important class of hybrid systems. This family of systems is generally modeled by a set of linear systems with transitions between models that are determined by a Markov chain taking values in a finite set. Markovian jump systems are popular in modeling many practical systems where one may experience abrupt changes in the system structure and parameters. These changes are quite common and do frequently occur in manufacturing systems, power systems, etc. Recently, MJLS's have also gained interest for their capability in modeling behaviors and phenomenon in networks that are manifested among sensors, actuators and processors [10], [11]. Furthermore, the study of MJLSD's has received considerable interest during the past few years [12][14].

N. Meskin and K. Khorasani are with the Department of Electrical and Computer Engineering, Concordia University, 1455 de Maisonneuve Blvd. W., Montreal, Quebec, Canada, H3G 1M8, email:\{n_meskin,kash\}@ece.concordia.ca. This research is supported in part by Strategic Projects and Discovery grants from the Natural Sciences and Engineering Research Council of Canada (NSERC).
In recent years, only a few work on FDI of MJLS's have appeared in the literature. In [15]-[18], a robust fault detection (and not an isolation) filter for MJLS's is developed based on an $H_{\infty}$ filtering framework, in which the residual generator is also an MJLS. A linear matrix inequality (LMI) approach is developed for solving the problem. In [19], a robust fault identification filter for a class of discrete-time MJLS's with mode dependent time-delays and norm bounded uncertainty is developed based on an $H_{\infty}$ optimization technique where the generated residual signal is an estimate of the fault signal. In [20], [21], the FDI problem for discrete-time MJLS's is solved based on a geometric framework. However, the problem of fault isolation for continuous-time MJLS's with and without time-delays has not been completely solved and fully addressed in the above references.

In this paper, we have adopted a geometric approach to the FDI problem of MJLS's. Towards this end, the first contribution of this paper is in the derivation of a geometric property for the unobservable subspace of MJLS's (Theorem 3.3) based on the notion of weak observability that was introduced recently in [22] and the development of a new approach for determining the weak-observability (Algorithm 1). It should be pointed out that recently in [9], invariant subspaces for discrete-time infinite MJLS's is introduced for defining detectability of these systems. However, in our work the stronger notion of weak observability is utilized for introducing the concept of unobservability subspaces.

The notion of a finite unobservable subspace for the MJLSD's is subsequently introduced and its geometric properties are investigated. It is shown that there exist similarities between the unobservable subspaces in the MJLS's and the MJLSD's. This similarity leads us to introduce the notion of an unobservability subspace for both systems. To propose an algorithm for obtaining this subspace, an alternative definition of an unobservability subspace is introduced in Theorem 4.5 which only depends on the matrices of the system. Based on this alternative definition, an algorithm for constructing the smallest unobservability subspace containing a given subspace is proposed (Algorithm 3). It should be noted that our proposed unobservability subspace is the most general concept of its own and all the previously developed unobservability subspaces for linear [2], LPV [23], bilinear [24], time-delay systems [25], [26] and the MJLS's with irreducible Markov process [27] can be considered as a special case of our proposed unobservability subspace.

By utilizing the developed geometric framework, necessary and sufficient conditions for solving the fundamental problem in residual generation (FPRG) [2] are derived for the MJLS's (Theorem 5.2). Moreover, sufficient conditions for solving the FPRG are derived for the MJLSD's (Theorem 5.3). Finally, 
we investigate the problem of designing an $H_{\infty}$-based FDI algorithm for an MJLS that has an unknown transition matrix and is subjected to external disturbances. These results are obtained by integrating our proposed geometric approach with an $H_{\infty}$ disturbance attenuation technique (Theorem 6.3). To summarize, the contributions of this work are as follows:

1) Derivation of a geometric property of an unobservable subspace for the MJLS's.

2) Development of a finite unobservable subspace for the MJLSD's.

3) Introducing the notion of unobservability subspace for the first time in the literature for continuous-time MJLS's and MJLSD's.

4) Derivation of necessary and sufficient conditions for solving the FPRG for the MJLS's.

5) Derivation of sufficient conditions for solving the FPRG for the MJLSD's.

6) Development of a robust fault detection and isolation algorithm for the MJLS's.

The remainder of this paper is organized as follows. In Section II, a brief background on geometric properties of linear systems as well as Markov processes is provided. In Section III-A, geometric characteristics of unobservable subspaces for the MJLS's are developed. The notion of a finite unobservable subspace for the MJLSD's is then introduced in Section III-B. In Section IV, an unobservability subspace is introduced formally for both the MJLS's and MJLSD's. In Section V, the solvability conditions for the FPRG for the MJLS's and the MJLSD's are obtained. An $H_{\infty}$-based fault detection and isolation strategy for the MJLS's is developed in Section VI. Conclusions and future work are presented in Section VII.

The following notation is used throughout the paper. Script letters $\mathcal{X}, \mathcal{U}, \mathcal{Y}, \ldots$, denote real vector spaces. $\mathcal{B}=\operatorname{Im} B$ denotes the image of $B$ and $\operatorname{Ker} C$ denotes the kernel of $C$. We say a map $C: \mathcal{X} \rightarrow \mathcal{Y}$ is monic if $\operatorname{Ker} C=0$ and is epic if $\operatorname{Im} C=\mathcal{Y}$. If a map $C$ is epic, then $C^{-r}$ denotes a right inverse of $C$ (i.e., $C C^{-r}=I$ ). For any positive integer $\mathbf{k}, \mathbf{k}$ denotes the finite set $\{1,2, \cdots, k\}$. The cardinality of a set $\mathrm{N}$ is denoted by $|\mathrm{N}|$. A subspace $\mathcal{S} \subseteq \mathcal{X}$ is termed $A$ invariant if $A \mathcal{S} \subseteq \mathcal{S}$. For an $A$-invariant subspace $\mathcal{S} \subseteq \mathcal{X}$, $A: \mathcal{S}$ denotes the restriction of $A$ to $\mathcal{S}$, and $A: \mathcal{X} / \mathcal{S}$ denotes the map induced by $A$ on the factor space $\mathcal{X} / \mathcal{S}$. For a linear system $(C, A, B),<\operatorname{Ker} C \mid A>$ denotes the unobservable subspace of $(C, A)$. For a given subspace $\mathcal{L}, \operatorname{dim}(\mathcal{L})$ denotes the dimension of $\mathcal{L}$. For given maps $A_{i}, i \in \Psi$ and a subspace $\mathcal{K}$, the largest $A_{i}$-invariant subspace $i \in \Psi$ that is contained in $\mathcal{K}$ is denoted by $\langle<\mathcal{K}| A_{i}>_{i \in \Psi}$. We denote by $\|$.$\| the$ standard norm in $\mathbb{R}^{n} . \mathscr{L}_{2}[0, \infty]$ stands for the space of square integrable vector functions over $[0, \infty)$. For $d(t) \in \mathscr{L}_{2}[0, \infty]$, $\|d\|_{2}$ denotes the $\mathscr{L}_{2}$-norm of $d(t)$. The asterisk $(*)$ is used to denote a matrix which can be inferred by symmetry. It is assumed throughout the paper that the reader is familiar with basic geometric concepts for analysis and design of linear systems (refer to [28] and [29]).

\section{BACKGROUND}

\section{A. Geometric FDI Approach}

Consider the linear system $\dot{x}(t)=A x(t), y(t)=C x(t)$, where $x \in \mathcal{X}$ is the state of the system and $y \in \mathcal{Y}$ is the output signal.

Definition 1: A subspace $\mathcal{W} \subseteq \mathcal{X}$ is said to be $(C, A)$ invariant (conditioned invariant) if $A(\mathcal{W} \cap \operatorname{Ker} C) \subseteq \mathcal{W}$.

It can be shown [29] that $\mathcal{W} \subseteq \mathcal{X}$ is $(C, A)$-invariant if and only if there exists an output injection map $D$ such that $(A+$ $D C) \mathcal{W} \subseteq \mathcal{W}$. In the geometrical approach to the FDI problem the concept of unobservability subspace plays a central role [2] as defined below.

Definition 2: A subspace $\mathcal{S}$ is an $(C, A)$ unobservability subspace (u.o.s.) [2] if $\mathcal{S}=<\operatorname{Ker} H C \mid A+D C>$ for some output injection map $D$ and measurement mixing map $H$.

It can be shown that for an unobservability subspace $\mathcal{S}$, $\mathcal{S}=<\mathcal{S}+\operatorname{Ker} C \mid A+D C>$. The next theorem provides an alternative characterization of the u.o.s. which is independent of the maps $D$ and $H$ (this is dual to Theorem 5.3 in [28] for controllability subspaces).

Theorem 2.1: Let $\mathcal{S} \subseteq \mathcal{X}$ and define the family $\mathcal{G}_{(A, C)}=$ $\left\{\mathscr{S} \mid \mathscr{S}=\mathcal{S}+A^{-1} \mathscr{S} \cap \operatorname{Ker} C\right\} . \mathcal{S}$ is said to be an $(C, A)$ u.o.s. if and only if $\mathcal{S}$ is conditioned invariant and $\mathcal{S}=\mathscr{S}^{*}$, where $\mathscr{S}^{*}$ is the maximal element of $\mathcal{G}_{(A, C)}$.

The family of u.o.s. that is containing $\mathcal{L} \subseteq \mathcal{X}$ is closed under intersection and is nonempty; therefore, it contains an infimal element $\mathcal{S}^{*}$. In [2], an algorithm for constructing $\mathcal{S}^{*}$ is proposed.

\section{B. Modes Classification of Markov Processes}

In this section, modes classification of continuous-time Markov processes is reviewed [30]. Consider a continuoustime Markov process $\{\lambda(t), t \geq 0\}$ taking values in the finite set $\Psi=\{1, \ldots, N\}$ with the following probability transitions:

$$
\mathbb{P}\{\lambda(t+h)=j \mid \lambda(t)=i\}= \begin{cases}\pi_{i j} h+o(h) & i \neq j \\ 1+\pi_{i i} h+o(h) & i=j\end{cases}
$$

where $\pi_{i j}$ is the transition rate from mode $i$ to mode $j$ with $\pi_{i j} \geq 0$ when $i \neq j, \pi_{i i}=-\sum_{j=1, j \neq i}^{N} \pi_{i j}$, and $o(h)$ is a function that satisfies $\lim _{h \rightarrow 0} \frac{o(h)}{h}=0$. Let $\Pi=\left[\pi_{i j}\right], i, j \in$ $\Psi$. The mode $j$ is accessible from mode $i$ (denoted by $i \rightsquigarrow j$ ) if there is a nonzero probability that the state $j$ can be reached from mode $i$ in some finite number of transitions. Moreover, the mode $j$ is said to be directly accessible from mode $j$ if $\pi_{i j}>0$. Modes $i$ and $j$ communicate (denoted by $i \rightsquigarrow j$ ) if both $i \rightsquigarrow j$ and $j \rightsquigarrow i$. A subset $\Phi \subseteq \Psi$ is a communication class of the Markov process $\lambda(t)$ if every pairs of modes in $\Phi$ communicate.

A communicating class $\Phi$ is closed if no mode outside the class is accessible from a mode in the class. A communicating class $\Phi$ is open if it is not closed; i.e. if there is a nonzero probability for the Markov process to leave $\Phi$. Hence, a Markov process can be partitioned into $\beta$ disjoint communicating classes $\Phi_{\alpha}, \alpha=1, \ldots, \beta$, where the first $\delta \leq \beta$ classes are closed and the remaining ones are open. Moreover, for an open communicating class $\Phi_{\alpha}, \alpha>\delta$, the set $\mathrm{N}_{\alpha}$ denotes the set of all classes that are accessible from modes 
in $\Phi_{\alpha}$. We also denote the set of all classes that are directly accessible from modes in $\Phi_{\alpha}$ as $\mathrm{N}_{\alpha}^{d}$. It follows that $\mathrm{N}_{\alpha}^{d} \subseteq \mathrm{N}_{\alpha}$. For a Markov process $\lambda(t)$ with $\beta$ classes, one can define a Markov process $\bar{\lambda}(t)$ taking values in $\bar{\Psi}=\{1, \ldots, \beta\}$, which represents jumps between the classes of $\lambda(t)$.

A Markov process transition matrix $\Pi$ is said to be in a canonical form if the modes are relabeled (re-ordered) so that states within the closed communication classes appear together first, followed by the modes in the open communication classes appear together. The open communicating classes are relabeled such that for each open communicating class $\Phi_{\alpha}$, none of the classes $\Phi_{j}, j \geq \alpha$ is accessible from modes in $\Phi_{\alpha}$. It follows that a canonical form of $\Pi$ is not unique and is a block lowertriangular matrix.

For an open class $\Phi_{\alpha}$, we denote $\mathrm{N}_{\alpha}^{o}$ and $\mathrm{N}_{\alpha}^{c}$ as the sets of open and closed classes, respectively, that are accessible from $\Phi_{\alpha}$. Furthermore, we set $\mathrm{N}_{\alpha}^{o}=\left\{\alpha_{1}^{o}, \ldots, \alpha_{\left|\mathrm{N}_{\alpha}\right|}^{o}\right\}$ and $\mathrm{N}_{\alpha}^{c}=$ $\left\{\alpha_{1}^{c}, \ldots, \alpha_{\left|N_{\alpha}^{c}\right|}^{c}\right\}$, where $\alpha_{1}^{c}<\alpha_{2}^{c}<\cdots<\alpha_{1}^{o}<\alpha_{2}^{o}<\cdots$. For each class $\Phi_{l}, l \in \mathrm{N}_{\alpha}$, we denote $\Gamma_{l}^{\alpha}$ as the set of all classes in $\mathrm{N}_{\alpha} \cup \alpha$ such that $\Phi_{l}$ is directly accessible from them. Moreover, we define $\Gamma_{l}^{\alpha^{*}} \subseteq \Gamma_{l}^{\alpha}$ as the set of classes $j \in \Gamma_{l}^{\alpha}$ such that they are not accessible from each other and if there exist $j, k \in \Gamma_{l}^{\alpha}$ such that $j \in \mathbf{N}_{k}$, then $j \in \Gamma_{l}^{\alpha^{*}}$ and $k \notin \Gamma_{l}^{\alpha^{*}}$. In other words, for constructing $\Gamma_{l}^{\alpha^{*}}$, if there exists a pair of classes $j, k \in \Gamma_{l}^{\alpha}$ such that $j \in \mathrm{N}_{k}$, then the class $k$ will not be included in $\Gamma_{l}^{\alpha^{*}}$. The following example shows how one can obtain a canonical form of $\Pi$.

Example 1: Consider a continuous-time Markov process on $\Psi=\{1,2,3,4,5,6,7\}$ with the following transition rate matrix $\Pi=\left[\begin{array}{ccccccc}-1 & 0 & 1 & 0 & 0 & 0 & 0 \\ 1 & -2 & 0 & 0 & 1 & 0 & 0 \\ 1 & 0 & -1 & 0 & 0 & 0 & 0 \\ 0 & 2 & 1 & -4 & 1 & 0 & 0 \\ 0 & 0 & 0 & 0 & 0 & 0 & 0 \\ 0 & 0 & 1 & 0 & 0 & -1 & 0 \\ 1 & 2 & 0 & 0 & 0 & 0 \\ 1 & 0 & 0 & 0\end{array}\right]$. It is clear that modes 1 and 3 form one closed communication class $\Phi_{1}$, the mode 5 forms the second closed class $\Phi_{2}$, and modes 2, 4, 6 and 7 form four disjoint open classes, where $\Phi_{3}=\{6\}, \Phi_{4}=\{2\}, \Phi_{5}=\{4\}$, and $\Phi_{6}=\{7\}$. By re-ordering the modes according to the sequence $1,3,5,6,2,4$, and 7 , the following canonical form of $\Pi$ can be obtained, namely $\Pi=\left[\begin{array}{ccccccc}-1 & 1 & 0 & 0 & 0 & 0 & 0 \\ 1 & -1 & 0 & 0 & 0 & 0 & 0 \\ 0 & 0 & 0 & 0 & 0 & 0 & 0 \\ 0 & 1 & 0 & -1 & 0 & 0 & 0 \\ 1 & 0 & 1 & 0 & -2 & 0 & 0 \\ 0 & 1 & 1 & 0 & 2 & -4 & 0 \\ 1 & 0 & 1 & 2 & 1 & 1 & -6\end{array}\right]$.

For instance, we have $\mathrm{N}_{6}=\{1,2,3,4,5\}, \Gamma_{1}^{6^{*}}=\{3,4\}$, $\Gamma_{1}^{6}=\{3,4,5,6\}, \Gamma_{2}^{6^{*}}=\{4\}$, and $\Gamma_{2}^{6}=\{4,5,6\}$. Moreover, the associated $\bar{\lambda}(t)$ for the above Markov process has 6 modes.

\section{UNOBSERVABLE SUbSPACE}

In this section, a geometric property of an unobservable subspace for the MJLS's is derived based on the notion of weak observability that was introduced in [22]. Moreover, the notion of finite unobservable subspace is introduced for the MJLSD's. This is a crucial step for defining the notion of unobservability subspaces for both the MJLS's and the MJLSD's. It will be shown subsequently that there exist similarities between the notion of finite unobservable subspace for the MJLSD's and the notion of unobservable subspace for the MJLS's. We will use these similarities in Section IV to introduce the notion of an unobservability subspace for the MJLS's and the MJLSD's.

\section{A. Unobservable Subspace for the MJLS's}

Consider the following Markovian jump linear system (MJLS)

$$
\begin{aligned}
& \dot{x}(t)=A_{\lambda(t)} x(t) \\
& y(t)=C_{\lambda(t)} x(t) \quad x(0)=x_{0}, \lambda(0)=i_{0}
\end{aligned}
$$

where $x \in \mathcal{X}$ is the continuous-time state of the system with dimension $n ; y \in \mathcal{Y}$ is the output with dimension $q$; and $\{\lambda(t), t \geq 0\}$ is a continuous-time Markov process taking values in the finite set $\Psi=\{1, \ldots, N\}$ as defined in Section II-B. The Markov process $\lambda(t)$ describes the switching between different system modes. It is assumed that matrix $\Pi$ is in a canonical form. The matrices $A_{\lambda(t)}$ and $C_{\lambda(t)}$ are known constant matrices for all $\lambda(t)=i \in \Psi$. For simplicity, we denote the matrices associated with $\lambda(t)=i$ by $A_{\lambda(t)}=A_{i}$ and $C_{\lambda(t)}=C_{i}$. Furthermore, the MJLS (1) is represented by $(\mathfrak{A}, \mathfrak{C}, \Pi)$, where $\mathfrak{A}=\left(A_{1}, \ldots, A_{N}\right)$ and $\mathfrak{C}=\left(C_{1}, \ldots, C_{N}\right)$. We first start with the definition of weak observability for the MJLS (1).

Definition 3 ( [22]): The system $(\mathfrak{A}, \mathfrak{C}, \Pi)$ is said to be weakly (W-) observable when there exist $t_{d} \geq 0$ and $\gamma>0$ such that $W^{t_{d}}(x, i) \geq \gamma|x|^{2}$ for each $x \in \mathcal{X}$ and $i \in \Psi$ where $W^{t}(x, i)$ is defined as

$$
\mathbb{E}\left\{\int_{0}^{t} x^{\prime}(\tau) C_{\lambda(\tau)}^{\prime} C_{\lambda(\tau)} x(\tau) d \tau \mid x(0)=x, \lambda(0)=i\right\}
$$

In [22], a collection of matrices $\mathcal{O}=\left\{\mathcal{O}_{1}, \ldots, \mathcal{O}_{N}\right\}$ is introduced for testing the W-observability of the MJLS (1) according to the following procedure. Let $O_{i}(0)=C_{i}^{\prime} C_{i}, i \in \Psi$, and define the sequence of matrices as

$$
O_{i}(k)=A_{i}^{\prime} O_{i}(k-1)+O_{i}(k-1) A_{i}+\sum_{j=1}^{N} \pi_{i j} O_{j}(k-1)
$$

where $k>0$ and $i \in \Psi$. The matrix $\mathcal{O}_{i}$ is then defined according to $\mathcal{O}_{i}=\left[O_{i}(0) O_{i}(1) \cdots O_{i}\left(n^{2} N-1\right)\right]^{\prime}$.

Theorem 3.1 ( [22]): The MJLS (1) is W-observable if and only if $\mathcal{O}_{i}$ has a full rank for each $i \in \Psi$.

By considering the above definition of W-observability, one can define the set of unobservable states for each mode $i$ as follows.

Definition 4: A state $(x, i)$ is said to be unobservable if $W^{t}(x, i)=0$ for all $t \geq 0$.

Let $\mathcal{Q}_{i}$ denote the mode $i$ unobservable ( $i$-unobservable) set of the MJLS (1), i.e. $\mathcal{Q}_{i}=\left\{x \in \mathcal{X} \mid W^{t}(x, i)=0, \forall t \geq\right.$ $0\}$. It is shown in [22] that $x \in \mathcal{N}\left\{\mathcal{O}_{i}\right\}$ and $W^{t}(x, i)=0$, for all $t \geq 0$ are equivalent. Hence, it follows that $\mathcal{Q}_{i}=$ $\mathcal{N}\left\{\mathcal{O}_{i}\right\}$. Therefore, $\mathcal{Q}_{i}$ is the subspace of $\mathcal{X}$ and is called the $i$-unobservable subspace of the MJLS (1). Next, we show that $\mathcal{Q}_{i}$ is $A_{i}$-invariant.

Lemma 3.2: The $i$-unobservable subspace is $A_{i}$-invariant, i.e. $A_{i} \mathcal{Q}_{i} \subseteq \mathcal{Q}_{i}$.

Proof: It is shown in [22] that if $i \rightsquigarrow j$, then $\mathcal{N}\left\{\mathcal{O}_{i}\right\} \subseteq$ $\mathcal{N}\left\{\mathcal{O}_{j}\right\}$. Hence, $\mathcal{Q}_{i} \subseteq \mathcal{N}\left\{\mathcal{O}_{j}\right\}$ for all modes $j$ such that $i \rightsquigarrow j$. Let $x \in \mathcal{Q}_{i}$. Our goal is to show that $A_{i} x \in \mathcal{Q}_{i}$. We have, 
$x \in \mathcal{N}\left\{O_{j}(k-1)\right\}$ and $x \in \mathcal{N}\left\{O_{j}(k)\right\}, \forall k$ and for all modes $j$ such that $i \rightsquigarrow j$. Therefore,

$$
\begin{aligned}
O_{i}(k) x= & A_{i}^{\prime} O_{i}(k-1) x+O_{i}(k-1) A_{i} x+ \\
& \sum_{j=1}^{N} \pi_{i j} O_{j}(k-1) x=O_{i}(k-1) A_{i} x=0
\end{aligned}
$$

since $O_{i}(k-1) x=O_{i}(k) x=0\left(x \in \mathcal{Q}_{i}\right)$ and for all $j$ such that $\pi_{i j} \neq 0$, we have $i \rightsquigarrow j$ and $O_{j}(k-1) x=0$. Hence, $A_{i} x \in \mathcal{N}\left\{O_{i}(k-1)\right\}$ for all $k$ and $\mathcal{Q}_{i}$ is $A_{i}$-invariant.

As shown in [22] if $i \leadsto j$, then $\mathcal{N}\left\{\mathcal{O}_{i}\right\}=\mathcal{N}\left\{\mathcal{O}_{j}\right\}$, and hence $\mathcal{Q}_{i}=\mathcal{Q}_{j}$. In other words, the unobservable subspaces of all modes in a communicating class are identical. Therefore, for each class $\Phi_{\alpha}$, we denote $\mathcal{Q}_{\alpha}$ as the unobservable subspace of all modes in $\Phi_{\alpha}$. The next theorem characterizes a geometric property of $\mathcal{Q}_{\alpha}$.

Theorem 3.3: For a class $\Phi_{\alpha}, \mathcal{Q}_{\alpha}$ is the largest $A_{i}$-invariant subspace $\left(i \in \Phi_{\alpha}\right)$ that is contained in $\bigcap_{i \in \Phi_{\alpha}} \operatorname{Ker} C_{i} \cap \mathcal{K}_{\alpha}$, i.e. $\mathcal{Q}_{\alpha}=<<\bigcap_{i \in \Phi_{\alpha}} \operatorname{Ker} C_{i} \bigcap \mathcal{K}_{\alpha} \mid A_{i}>>_{i \in \Phi_{\alpha}}$ where if $\Phi_{\alpha}$ is closed then $\mathcal{K}_{\alpha}=\mathcal{X}$, and if $\Phi_{\alpha}$ is open then $\mathcal{K}_{\alpha}=\bigcap_{l \in \mathrm{N}^{d}} \mathcal{Q}_{l}$. Proof: Consider a class $\Phi_{\alpha}$ where we have $\mathcal{Q}_{\alpha}=\mathcal{N}\left\{\mathcal{O}_{i}\right\}$, $i \in \Phi_{\alpha}$. It is clear that $\mathcal{Q}_{\alpha} \subseteq \operatorname{Ker} C_{i}, i \in \Phi_{\alpha}$, and hence $\mathcal{Q}_{\alpha} \subseteq \bigcap_{i \in \Phi_{\alpha}} \operatorname{Ker}_{i}$. Moreover, according to Lemma 3.2 and the fact that $\mathcal{Q}_{i}=\mathcal{Q}_{j}=\mathcal{Q}_{\alpha}$ for all $i, j \in \Phi_{\alpha}, \mathcal{Q}_{\alpha}$ is $A_{i}$ invariant, $i \in \Phi_{\alpha}$.

First assume that $\Phi_{\alpha}$ is closed. We show that $\mathcal{Q}_{\alpha}=<<$ $\bigcap_{i \in \Phi_{\alpha}} \operatorname{Ker} C_{i} \mid A_{i}>>_{i \in \Phi_{\alpha}}$. Let $\mathcal{V}$ be an $A_{i}$-invariant subspace $\left(i \in \Phi_{\alpha}\right)$ that is contained in $\bigcap_{i \in \Phi_{\alpha}} \operatorname{Ker} C_{i}$. Clearly, $\mathcal{V} \subseteq$ $\operatorname{Ker} C_{i}=\mathcal{N}\left\{O_{i}(0)\right\}, i \in \Phi_{\alpha}$. Let $\mathcal{V} \subseteq \mathcal{N}\left\{O_{i}(k-1)\right\}, i \in \Phi_{\alpha}$ and $x \in \mathcal{V}$, then

$$
\begin{aligned}
O_{i}(k) x= & A_{i}^{\prime} O_{i}(k-1) x+O_{i}(k-1) A_{i} x+ \\
& \sum_{i=1}^{N} \pi_{i j} O_{j}(k-1) x=0
\end{aligned}
$$

since $A_{i} x \in \mathcal{V}\left(\mathcal{V}\right.$ is $A_{i}$-invariant) and for all $j$ such that $\pi_{i j} \neq 0$, we have $j \in \Phi_{\alpha}\left(\Phi_{\alpha}\right.$ is closed) and $O_{j}(k-1) x=0$. Hence, $x \in \mathcal{N}\left\{O_{i}(k)\right\}, i \in \Phi_{\alpha}$ for all $k$ and $\mathcal{V} \subseteq \mathcal{Q}_{\alpha}$. This shows that $\mathcal{Q}_{\alpha}$ contains all the subspaces that are $A_{i}$-invariant $\left(i \in \Phi_{\alpha}\right)$ and is contained in $\bigcap_{i \in \Phi_{\alpha}} \operatorname{Ker} C_{i}$.

Now if $\Phi_{\alpha}$ is an open class, then for all modes that are accessible from modes in $\Phi_{\alpha}$, i.e. $\forall j \in \Phi_{l}, l \in \mathrm{N}_{\alpha}$, we have $\mathcal{Q}_{\alpha} \subseteq \mathcal{N}\left\{\mathcal{O}_{j}\right\}=\mathcal{Q}_{l}$. Moreover, we have $\bigcap_{l \in \mathrm{N}_{\alpha}} \mathcal{Q}_{l}=$ $\bigcap_{l \in \mathrm{N}_{\alpha}^{d}} \mathcal{Q}_{l}$, since if there exists a class $j \in \mathrm{N}_{\alpha}$ and $j \notin \mathrm{N}_{\alpha}^{d}$, there must exist a class $\Phi_{k}, k \in \mathrm{N}_{\alpha}^{d}$ such that $j \in \mathrm{N}_{k}$ which leads to $\mathcal{Q}_{k} \subseteq \mathcal{Q}_{j}$. Hence, $\mathcal{Q}_{\alpha} \subseteq \bigcap_{i \in \Phi_{\alpha}} \operatorname{Ker}_{i} \bigcap_{l \in \mathrm{N}_{\alpha}^{d}} \mathcal{Q}_{l}$. Next, we show that $\mathcal{Q}_{\alpha}$ is the largest $A_{i}$-invariant $i \in \Phi_{\alpha}^{\alpha}$ that is contained in $\bigcap_{i \in \Phi_{\alpha}} \operatorname{Ker} C_{i} \bigcap \mathcal{K}_{\alpha}$. Let $\mathcal{V}$ be an $A_{i}$-invariant $\left(i \in \Phi_{\alpha}\right)$ subspace that is contained in $\bigcap_{i \in \Phi_{\alpha}} \operatorname{Ker} C_{i} \bigcap \mathcal{K}_{\alpha}$. Clearly, $\mathcal{V} \subseteq \operatorname{Ker} C_{i}=\mathcal{N}\left\{O_{i}(0)\right\}, i \in \Phi_{\alpha}$, and $\mathcal{V} \subseteq$ $\mathcal{N}\left\{O_{j}(k)\right\}$ for all $k \geq 0$ and $j \in \Phi_{l}, l \in \mathrm{N}_{\alpha}^{d}$. Let $\mathcal{V} \subseteq \mathcal{N}\left\{O_{i}(k-1)\right\}, i \in \Phi_{\alpha}$ and $x \in \mathcal{V}$, then $O_{i}(k) x=$ $A_{i}^{\prime} O_{i}(k-1) x+O_{i}(k-1) A_{i} x+\sum_{i=1}^{N} \pi_{i j} O_{j}(k-1) x=0$, since $A_{i} x \in \mathcal{V}$ and for all $j$ such that $\pi_{i j} \neq 0$, whether $j \in \Phi_{\alpha}$ or $j \in \Phi_{l}, l \in \mathrm{N}_{\alpha}^{d}$ we have $O_{j}(k-1) x=0$. Hence, $\mathcal{V} \subseteq \mathcal{N}\left\{O_{i}(k)\right\}$ for all $k$ and $\mathcal{V} \subseteq \mathcal{Q}_{\alpha}$.

Motivated from Theorem 3.3, the following algorithm provides a procedure for constructing the required subspace $\mathcal{Q}_{\alpha}$.
Algorithm 1: The subspace $\mathcal{Q}_{\alpha}$ is obtained from the sequence $\mathcal{Z}_{0}=\bigcap_{i \in \Phi_{\alpha}} \operatorname{Ker} C_{i} \bigcap \mathcal{K}_{\alpha}$ with $\mathcal{Z}_{\mu}=\bigcap_{i \in \Phi_{\alpha}} \operatorname{Ker} C_{i} \bigcap \mathcal{K}_{\alpha} \bigcap_{j \in \Phi_{\alpha}} A_{j}^{-1} \mathcal{Z}_{\mu-1}$ such that whenever $\mathcal{Z}_{\mu}=\mathcal{Z}_{\mu+1}$, then $\mathcal{Z}_{\mu}=\mathcal{Q}_{\alpha}$.

\section{B. Unobservable Subspace for the MJLSD's}

In this section, we introduce the notion of finite unobservable subspace for the Markovian jump linear systems with time-delays (MJLSD's). Consider the following MJLSD

$$
\begin{aligned}
\dot{x}(t) & =A_{\lambda(t)} x(t)+A_{\lambda(t)}^{\tau} x\left(t-\tau_{\lambda(t)}(t)\right) \\
y(t) & =C_{\lambda(t)} x(t) \quad x(\theta)=\phi(\theta), \quad \theta \in[-\mu, 0]
\end{aligned}
$$

where $x \in \mathcal{X}$ is the continuous-time state of the system with dimension $n, y \in \mathcal{Y}$ is the output with dimension $q$, and $\mu=\max _{i, t}\left\{\tau_{i}(t)\right\}$. We denote the matrices $A_{\lambda(t)}^{\tau}$ that are associated with $\lambda(t)=i$ by $A_{i}^{\tau}$. Furthermore, the MJLSD (2) is represented by $\left(\mathfrak{A}, \mathfrak{A}^{\tau}, \mathfrak{C}, \Pi\right)$, where $\mathfrak{A}^{\tau}=\left(A_{1}^{\tau}, \ldots, A_{N}^{\tau}\right)$. In system $(2), \tau_{\lambda(t)}(t)$ denotes a time-varying delay for the mode $\lambda(t)$.

Since the behavior of system (2) in each mode is governed by the retarded time-delay equation, we need to first define an unobservable subspace for each mode of the system. Recently, the notion of finite unobservable subspace is introduced for retarded time-delay systems in [25]. Consider the following time-delay system that corresponds to the $i$-th mode of the MJLSD (2), namely

$$
\begin{aligned}
& \dot{x}(t)=A_{i} x(t)+A_{i}^{\tau} x\left(t-\tau_{i}(t)\right) \\
& y(t)=C_{i} x(t), \quad x(\theta)=\phi(\theta), \quad \theta \in[-\mu, 0]
\end{aligned}
$$

Definition 5 ( [25]): A finite unobservable subspace (denoted by $\left.\mathcal{Q}^{\tau i}\right)$ for system (3) is defined as the largest $\left(A_{i}\right.$, $A_{i}^{\tau}$ )-invariant subspace contained in $\operatorname{Ker} C_{i}$.

It is shown in [25] that for all $x \in \mathcal{Q}^{\tau i}$, we have $y(t)=0$. Based on the above definition and the results of Section III-B, we formally define the notion of finite unobservable subspace $\mathcal{Q}_{\alpha}^{\tau}$ for each class of the MJLSD (2) as follows.

Definition 6: For a class $\Phi_{\alpha}, \mathcal{Q}_{\alpha}^{\tau}$ is the largest $\left(A_{i}, A_{i}^{\tau}\right)$-invariant subspace $\left(i \in \Phi_{\alpha}\right)$ that is contained in $\bigcap_{j \in \Phi_{\alpha}} \operatorname{Ker}_{j} \cap \mathcal{K}_{\alpha}$, i.e. $\quad \mathcal{Q}_{\alpha}^{\tau}=<<$ $\bigcap_{j \in \Phi_{\alpha}} \operatorname{Ker} C_{j} \bigcap \mathcal{K}_{\alpha} \mid A_{i}, A_{i}^{\tau}>>_{i \in \Phi_{\alpha}}$, where if $\Phi_{\alpha}$ is closed then $\mathcal{K}_{\alpha}=\mathcal{X}$, and if $\Phi_{\alpha}$ is open then $\mathcal{K}_{\alpha}=\bigcap_{l \in \mathrm{N}_{\alpha}^{d}} \mathcal{Q}_{l}^{\tau}$.

It is clear from Definition 6 that if $x \in \mathcal{Q}_{\alpha}^{\tau}$ and $i \in \Phi_{\alpha}$, then $W^{t}(x, i)=0$. Moreover, according to Theorem 3.3 and Definition 6, $\mathcal{Q}_{\alpha}$ and $\mathcal{Q}_{\alpha}^{\tau}$ are the largest finite $A_{i}$-invariant and $\left(A_{i}, A_{i}^{\tau}\right)$-invariant subspaces, respectively, that are contained in $\bigcap_{j \in \Phi_{\alpha}} \operatorname{Ker} C_{j} \cap \mathcal{K}_{\alpha}$. In the next section, we will use this similarity to develop the concept of unobservability subspaces for both the MJLS's and the MJLSD's.

\section{UNOBSERVABILITY SUBSPACE}

The concept of an unobservability subspace was used in [2] to solve the fundamental problem of residual generation (FPRG) for linear systems. In [31], the similar notion of observability codistribution is introduced for nonlinear systems and is shown that it can be used to address the nonlinear fundamental problem of residual generation ( $l \mathrm{NLFPRG})$. Similar 
problems are also considered for LPV and bilinear systems in [23] and [24], respectively.

In this section, the notion of unobservability subspace is formally introduced for both the MJLS's and the MJLSD's. To develop an algorithm for constructing this subspace, an alternative definition of unobservability subspace is presented, which only depends on the matrices of the system. Based on this alternative definition, an algorithm for constructing the smallest u.o.s. containing a given subspace is proposed. As shown in Section V, unobservability subspaces play a central role in solving the fault detection and isolation (FDI) problem for the Markovian jump systems.

\section{A. Unobservability Subspace for the MJLS's}

In this section, the notion of unobservability subspace for the MJLS's is formally introduced. We start by defining the notion of conditioned invariant subspace for a class $\Phi_{\alpha}$.

Definition 7: A subspace $\mathcal{W}_{\alpha}$ is said to be conditioned invariant for all the modes $i \in \Phi_{\alpha}$ of $(\mathfrak{A}, \mathfrak{C}, \Pi)$ if $A_{i}\left(\mathcal{W}_{\alpha} \cap\right.$ $\left.\operatorname{Ker} C_{i}\right) \subseteq \mathcal{W}_{\alpha}, i \in \Phi_{\alpha}$.

It is clear that if $\mathcal{W}_{\alpha}$ is conditioned invariant for $(\mathfrak{A}, \mathfrak{C}, \Pi)$, then $\mathcal{W}_{\alpha}$ is $\left(C_{i}, A_{i}\right)$-invariant for all $i \in \Phi_{\alpha}$. Therefore, there exist maps $D_{i}$ such that $\left(A_{i}+D_{i} C_{i}\right) \mathcal{W}_{\alpha} \subseteq \mathcal{W}_{\alpha}, i \in \Phi_{\alpha}$. One can also define the notion of conditioned invariant for more than one class of $(\mathfrak{A}, \mathfrak{C}, \Pi)$. Let $\Gamma$ be the subset of $\{1, \ldots, \beta\}$ which represents a subset of classes of $\lambda(t)$. A subspace $\mathcal{W}_{\Gamma}$ is then said to be conditioned invariant for $\Gamma$ if it is conditioned invariant for each class in $\Gamma$, i.e. $A_{i}\left(\mathcal{W}_{\Gamma} \cap \operatorname{Ker} C_{i}\right) \subseteq \mathcal{W}_{\Gamma}$, $i \in \Phi_{l}, l \in \Gamma$.

We denote the families of conditioned invariant subspaces for a class $\Phi_{\alpha}$ and for a subset of classes $\Gamma$ of $(\mathfrak{A}, \mathfrak{C}, \Pi)$ by $\mathfrak{W}_{\{\alpha\}}(\mathfrak{A}, \mathfrak{C})$ and $\mathfrak{W}_{\Gamma}(\mathfrak{A}, \mathfrak{C})$, respectively. It is clear that for $\Gamma=\{\alpha\}$, these two families are identical. Hence, we will focus on the more general family, i.e. $\mathfrak{W}_{\Gamma}(\mathfrak{A}, \mathfrak{C})$. If $\mathcal{W}_{\Gamma} \in \mathfrak{W}_{\Gamma}(\mathfrak{A}, \mathfrak{C})$, we write $\mathfrak{D}_{\Gamma}\left(\mathcal{W}_{\Gamma}\right)$ for the family of maps $D_{i}$ where $\left(A_{i}+D_{i} C_{i}\right) \mathcal{W}_{\Gamma} \subseteq \mathcal{W}_{\Gamma}, i \in \Phi_{l}, l \in \Gamma$. The notion of conditioned invariant subspace for $(\mathfrak{A}, \mathfrak{C}, \Pi)$ is a dual to that of the robust maximal controlled invariant which is introduced in [32]. By duality it can be shown that $\mathfrak{W}_{\Gamma}(\mathfrak{A}, \mathfrak{C})$ is closed under the operation of subspace intersection and is nonempty, and consequently for any given subspace $\mathcal{L} \subseteq \mathcal{X}$, the family of conditioned invariant subspaces that contains $\mathcal{L}$ (denoted by $\mathfrak{W}_{\Gamma}(\mathfrak{A}, \mathfrak{C}, \mathcal{L})$ ) has the infimal element which is denoted by $\mathcal{W}_{\Gamma}^{*}=\inf \mathfrak{W}_{\Gamma}(\mathfrak{A}, \mathfrak{C}, \mathcal{L})$. The following algorithm can be invoked for constructing $\mathcal{W}_{\Gamma}^{*}$ :

Algorithm 2: The subspace $\mathcal{W}_{\Gamma}^{*}$ is obtained from the sequence $\mathcal{W}_{k}=\mathcal{L}+\sum_{l \in \Gamma} \sum_{i \in \Phi_{l}} A_{i}\left(\mathcal{W}_{k-1} \cap \operatorname{Ker} C_{i}\right)$ with $\mathcal{W}_{0}=\mathcal{L}$ such that whenever $\mathcal{W}_{k}=\mathcal{W}_{k+1}$, then $\mathcal{W}_{\Gamma}^{*}=\mathcal{W}_{k}$.

Definition 8: A subspace $\mathcal{S}_{\alpha}$ is an unobservability subspace for all the modes $i \in \Phi_{\alpha}$ of $(\mathfrak{A}, \mathfrak{C}, \Pi)$ if there exist output injection maps $D_{i}$ and measurement mixing maps $H_{i}$ such that $\mathcal{S}_{\alpha}$ is an unobservable subspace of class $\Phi_{\alpha}$ of $\operatorname{system}(\tilde{\mathfrak{A}}, \tilde{\mathfrak{C}}, \Pi)$, where $\tilde{\mathfrak{A}}=\left\{A_{1}+D_{1} C_{1}, \ldots, A_{N}+D_{N} C_{N}\right\}$ and $\tilde{\mathfrak{C}}=\left\{H_{1} C_{1}, \ldots, H_{N} C_{N}\right\}$.

It follows that $\mathcal{S}_{\alpha}=<<\bigcap_{j \in \Phi_{\alpha}} \operatorname{Ker} H_{j} C_{j} \bigcap \mathcal{K}_{\alpha} \mid A_{i}+$ $D_{i} C_{i}>>_{i \in \Phi_{\alpha}}$, where if $\Phi_{\alpha}$ is closed, then $\mathcal{K}_{\alpha}=\mathcal{X}$, and if $\Phi_{\alpha}$ is open, then $\mathcal{K}_{\alpha}=\bigcap_{l \in \mathrm{N}_{\alpha}^{d}} \mathcal{S}_{l}$, where $\mathcal{S}_{l}$ is an u.o.s. for class $\Phi_{l}$.
Remark 1: It should be noted that the notion of unobservability subspace in Definition 8 is the most general concept of u.o.s. introduced in the literature and the ones that have been presented for linear [2], LPV [23], bilinear [24], and timedelay [25], [26] systems can be considered as a special case of our proposed unobservability subspace. Indeed, for $C_{i}=C$, $i \in \Phi_{\alpha}$, (identical output measurements) and $\mathcal{K}=\mathcal{X}$, the above definition coincides with the unobservability subspace for the LPV and the bilinear systems as stated in [23] and [24], respectively.

We denote the class of all unobservability subspaces in $\mathcal{X}$ for $(\mathfrak{A}, \mathfrak{C}, \Pi)$ by $\mathfrak{S}_{\alpha}(\mathfrak{A}, \mathfrak{C})$. In the following, our goal is to derive an alternative characterization for the unobservability subspace which is independent of the maps $D_{i}$ and $H_{i}$ as used in Definition 8 (the idea is similar to that in Theorem 2.1). As shown subsequently, this alternative definition provides us with means to obtain the unobservability subspaces more readily. The following lemma presents a result that is necessary for formulating our alternative definition.

Lemma 4.1: Let $\hat{\mathcal{S}}_{j} \subseteq \mathcal{X}$ such that $\operatorname{Ker} C_{j} \subseteq \hat{\mathcal{S}}_{j}, j \in$ $\Phi_{\alpha}$ and $<<\bigcap_{j \in \Phi_{\alpha}} \hat{\mathcal{S}}_{j} \bigcap \mathcal{K}_{\alpha} \mid A_{i}>>_{i \in \Phi_{\alpha}}=\mathcal{S}_{\alpha}$, then $<<$ $\bigcap_{j \in \Phi_{\alpha}}\left(\mathcal{S}_{\alpha}+\operatorname{Ker} C_{j}\right) \cap \mathcal{K}_{\alpha} \mid A_{i}>>_{i \in \Phi_{\alpha}}=\mathcal{S}_{\alpha}$. Conversely, if $<<\bigcap_{j \in \Phi_{\alpha}}\left(\mathcal{S}_{\alpha}+\operatorname{Ker} C_{j}\right) \bigcap \mathcal{K}_{\alpha} \mid A_{i}>>_{i \in \Phi_{\alpha}}=\mathcal{S}_{\alpha}$, then there exist maps $H_{j}, j \in \Phi_{\alpha}$ such that

$$
<<\bigcap_{j \in \Phi_{\alpha}} \operatorname{Ker}_{j} C_{j} \bigcap \mathcal{K}_{\alpha} \mid A_{i}>>_{i \in \Phi_{\alpha}}=\mathcal{S}_{\alpha}
$$

Proof: We have $\mathcal{S}_{\alpha} \subseteq \bigcap_{i \in \Phi_{\alpha}} \hat{\mathcal{S}}_{j} \cap \mathcal{K}_{\alpha} \subseteq \hat{\mathcal{S}}_{j}$ and $\operatorname{Ker} C_{j} \subseteq$ $\hat{\mathcal{S}}_{j}, \quad j \in \Phi_{\alpha}$, so that $\bigcap_{j \in \Phi_{\alpha}}\left(\mathcal{S}_{\alpha}+\operatorname{Ker} C_{j}\right) \cap \mathcal{K}_{\alpha} \subseteq$ $\bigcap_{j \in \Phi_{\alpha}} \hat{\mathcal{S}}_{j} \cap \mathcal{K}_{\alpha}$. Consequently,

$$
\begin{aligned}
& <<\bigcap_{j \in \Phi_{\alpha}}\left(\mathcal{S}_{\alpha}+\operatorname{Ker} C_{j}\right) \bigcap \mathcal{K}_{\alpha} \mid A_{i}>_{i \in \Phi_{\alpha}} \\
& \subseteq<<\bigcap_{j \in \Phi_{\alpha}} \hat{\mathcal{S}}_{j} \bigcap \mathcal{K}_{\alpha} \mid A_{i}>_{i \in \Phi_{\alpha}}=\mathcal{S}_{\alpha}
\end{aligned}
$$

On the other hand, $A_{i} \mathcal{S}_{\alpha} \subseteq \mathcal{S}_{\alpha}, \mathcal{S}_{\alpha} \subseteq \mathcal{K}_{\alpha}$, and

$$
\bigcap_{j \in \Phi_{\alpha}}\left(\mathcal{S}_{\alpha}+\operatorname{Ker} C_{j}\right) \supseteq \mathcal{S}_{\alpha}+\bigcap_{j \in \Phi_{\alpha}} \operatorname{Ker} C_{j} \supseteq \mathcal{S}_{\alpha}
$$

Hence, $\mathcal{S}_{\alpha} \subseteq<<\bigcap_{j \in \Phi_{\alpha}}\left(\mathcal{S}_{\alpha}+\operatorname{Ker} C_{j}\right) \bigcap \mathcal{K}_{\alpha} \mid A_{i}>>_{i \in \Phi_{\alpha}}$, and consequently

$$
\mathcal{S}_{\alpha}=<<\bigcap_{j \in \Phi_{\alpha}}\left(\mathcal{S}_{\alpha}+\operatorname{Ker} C_{j}\right) \bigcap \mathcal{K}_{\alpha} \mid A_{i}>>_{i \in \Phi_{\alpha}}
$$

To show the converse part, let $\left\{c_{1}^{j}, \ldots, c_{r}^{j}\right\}$ be a basis for $\mathcal{S}_{\alpha}+\operatorname{Ker} C_{j}$ such that $\left\{c_{r-p_{j}+1}^{j}, \ldots, c_{r}^{j}\right\}$ is the basis for $\operatorname{Ker} C_{j}$ $\left(\operatorname{dim}\left(\operatorname{Ker} C_{j}\right)=p_{j}\right)$. Therefore, $y_{i j}=C_{j} c_{i}^{j}, i=1, \ldots, r-p_{j}$ are independent. Let $\left\{y_{i 1}, \ldots, y_{i q}\right\}$ be a basis for $\mathcal{Y}$, and define $H_{j} y_{i j}=0, i=1, \ldots, r-p_{j}$, and $H_{j} y_{i j}=y_{i j}, i=r-p_{j}+$ $1, \ldots, q$. Consequently, $\operatorname{Ker} C_{j}+\mathcal{S}_{\alpha}=\operatorname{Ker} H_{j} C_{j}$, and therefore $\bigcap_{j \in \Phi_{\alpha}}\left(\mathcal{S}_{\alpha}+\operatorname{Ker} C_{j}\right)=\bigcap_{j \in \Phi_{\alpha}} \operatorname{Ker} H_{j} C_{j}$.

We are now in a position to state our next result.

Theorem 4.2: $\mathcal{S}_{\alpha} \in \mathfrak{S}_{\alpha}(\mathfrak{A}, \mathfrak{C})$ if and only if there exist maps $D_{i}$ such that $\mathcal{S}_{\alpha}=<<\bigcap_{j \in \Phi_{\alpha}}\left(\mathcal{S}_{\alpha}+\operatorname{Ker} C_{j}\right) \bigcap \mathcal{K}_{\alpha} \mid A_{i}+$ $D_{i} C_{i}>>_{i \in \Phi_{\alpha}}$.

Proof: The proof follows readily from Lemma 4.1 by taking $\hat{\mathcal{S}}_{j}=\operatorname{Ker} H_{j} C_{j}$. 
The above theorem eliminates the need for the maps $H_{i}$ from Definition 8. For a given u.o.s. $\mathcal{S}_{\alpha}$, the maps $H_{j}$ 's can be computed by solving the equations $\operatorname{Ker} H_{j} C_{j}=$ $\mathcal{S}_{\alpha}+\operatorname{Ker} C_{j}, i \in \Phi_{\alpha}$. Next, we try to the characterize the unobservability subspace with an algorithm that computes $\mathcal{S}_{\alpha}$ without explicitly constructing $D_{i}, i \in \Phi_{\alpha}$. For an arbitrary subspace $\mathcal{S}_{\alpha} \subseteq \mathcal{K}_{\alpha}$, let us define a family $\mathcal{G}_{(\mathfrak{A}, \mathfrak{C})}^{\alpha}=\{\mathscr{S} \mid \mathscr{S}=$ $\left.\bigcap_{i \in \Phi_{\alpha}}\left(\mathcal{S}_{\alpha}+\left(A_{i}^{-1} \mathscr{S} \cap \operatorname{Ker} C_{i}\right)\right) \bigcap \mathcal{K}_{\alpha}\right\}$. Below, we first show that $\mathcal{G}_{(\mathfrak{A}, \mathfrak{C})}^{\alpha}$ has a unique maximal member.

Lemma 4.3: There exists a unique element $\mathscr{S}^{*} \in \mathcal{G}_{(\mathfrak{A}, \mathfrak{C})}^{\alpha}$ such that $\mathscr{S} \subset \mathscr{S}^{*}, \forall \mathscr{S} \in \mathcal{G}_{(\mathfrak{A}, \mathfrak{C})}^{\alpha}$.

Proof: Define a sequence $\mathscr{S}^{\mu} \subseteq \mathcal{X}$ according to $\mathscr{S}^{0}=\mathcal{X}$, $\mathscr{S}^{\mu}=\bigcap_{i \in \Phi_{\alpha}}\left(\mathcal{S}_{\alpha}+\left(A_{i}^{-1} \mathscr{S}^{\mu-1} \cap \operatorname{Ker} C_{i}\right)\right) \bigcap \mathcal{K}_{\alpha}, \mu \in \mathbf{n}$. The sequence $\mathscr{S}^{\alpha}$ is non-increasing since $\mathscr{S}^{1} \subseteq \mathscr{S}^{0}$, and if $\mathscr{S}^{\mu} \subseteq$ $\mathscr{S}^{\mu-1}$, we have

$$
\begin{aligned}
\mathscr{S}^{\mu+1} & =\bigcap_{i \in \Phi_{\alpha}}\left(\mathcal{S}_{\alpha}+\left(A_{i}^{-1} \mathscr{S}^{\mu} \cap \operatorname{Ker} C_{i}\right)\right) \bigcap \mathcal{K}_{\alpha} \\
& \subseteq \bigcap_{i \in \Phi_{\alpha}}\left(\mathcal{S}_{\alpha}+\left(A_{i}^{-1} \mathscr{S}^{\mu-1} \cap \operatorname{Ker} C_{i}\right)\right) \bigcap \mathcal{K}_{\alpha}=\mathscr{S}^{\mu}
\end{aligned}
$$

Therefore, there exists a $k \leq n$ such that $\mathscr{S}^{k+1}=\mathscr{S}^{k}$, so that we set $\mathscr{S}^{*}=\mathscr{S}^{k}$. Clearly, $\mathscr{S}^{*} \in \mathcal{G}_{(\mathfrak{A}, \mathfrak{C})}$. Next, we show that $\mathscr{S}^{*}$ is the maximal element. Let $\mathscr{S} \in \mathcal{G}_{(\mathfrak{A}, \mathfrak{C})}^{\alpha}$, then $\mathscr{S} \subseteq \mathscr{S}^{0}$, and if $\mathscr{S} \subseteq \mathscr{S}^{\mu}$, we have

$$
\begin{aligned}
\mathscr{S} & =\bigcap_{i \in \Phi_{\alpha}}\left(\mathcal{S}_{\alpha}+\left(A_{i}^{-1} \mathscr{S} \cap \operatorname{Ker} C_{i}\right)\right) \bigcap \mathcal{K}_{\alpha} \\
& \subseteq \bigcap_{i \in \Phi_{\alpha}}\left(\mathcal{S}_{\alpha}+\left(A_{i}^{-1} \mathscr{S}^{\mu} \cap \operatorname{Ker} C_{i}\right)\right) \bigcap \mathcal{K}_{\alpha}=\mathscr{S}^{\mu+1}
\end{aligned}
$$

Consequently, $\mathscr{S} \subseteq \mathscr{S}^{\mu}$ for all $\mu$, and hence $\mathscr{S} \subseteq \mathscr{S}^{*}$.

The next lemma provides an important property of the maximal element $\mathscr{S}^{*}$ which will be used for introducing our suggested alternative characterization of the u.o.s. for system $(\mathfrak{A}, \mathfrak{C}, \Pi)$.

Lemma 4.4: Let $\mathcal{S}_{\alpha} \in \mathfrak{W}_{\{\alpha\}}(\mathfrak{A}, \mathfrak{C})$ and $D_{i} \in \mathfrak{D}_{\{\alpha\}}\left(\mathcal{S}_{\alpha}\right)$, then $\mathscr{S}^{*}$ is the largest $\left(A_{i}+D_{i} C_{i}\right)$-invariant $\left(i \in \Phi_{\alpha}\right)$ that is contained in $\bigcap_{j \in \Phi_{\alpha}}\left(\mathcal{S}_{\alpha}+\operatorname{Ker} C_{j}\right) \bigcap \mathcal{K}_{\alpha}$, i.e. $\mathscr{S}^{*}=<<$ $\bigcap_{j \in \Phi_{\alpha}}\left(\mathcal{S}_{\alpha}+\operatorname{Ker} C_{j}\right) \bigcap \mathcal{K}_{\alpha} \mid A_{i}+D_{i} C_{i}>>_{i \in \Phi_{\alpha}}$.

Proof: First we show that any $\mathscr{S} \in \mathcal{G}_{(\mathfrak{A}, \mathfrak{C})}^{\alpha}$ is $\left(A_{i}+D_{i} C_{i}\right)$ invariant $\left(i \in \Phi_{\alpha}\right)$. We have

$$
\begin{aligned}
\left(A_{j}+D_{j} C_{j}\right) \mathscr{S} & \subseteq\left(A_{j}+D_{j} C_{j}\right)\left(\mathcal{S}_{\alpha}+A_{j}^{-1} \mathscr{S} \cap \operatorname{Ker} C_{j}\right) \\
& \subseteq\left(A_{j}+D_{j} C_{j}\right) \mathcal{S}_{\alpha}+A_{j}\left(A_{j}^{-1} \mathscr{S} \cap \operatorname{Ker} C_{j}\right) \\
& \subseteq \mathcal{S}_{\alpha}+\mathscr{S} \subseteq \mathscr{S}
\end{aligned}
$$

where we used the relationship $\mathscr{S}=\bigcap_{i \in \Phi_{\alpha}}\left(\mathcal{S}_{\alpha}+\left(A_{i}^{-1} \mathscr{S} \cap\right.\right.$ $\left.\left.\operatorname{Ker} C_{i}\right)\right) \bigcap \mathcal{K}_{\alpha} \supseteq\left(\mathcal{S}_{\alpha}+\bigcap_{i \in \Phi_{\alpha}}\left(A_{i}^{-1} \mathscr{S} \cap \operatorname{Ker} C_{i}\right)\right) \bigcap \mathcal{K}_{\alpha} \supseteq \mathcal{S}_{\alpha}$ (since $\left.\mathcal{S}_{\alpha} \subseteq \mathcal{K}_{\alpha}\right)$ and $A_{j}\left(A_{j}^{-1} \mathscr{S}\right) \subseteq \mathscr{S}$. Therefore, $\left(A_{j}+\right.$ $\left.D_{j} C_{j}\right) \mathscr{S} \subseteq \mathscr{S}, j \in \Phi_{\alpha} ;$ and hence $\mathscr{S} \in \mathfrak{W}_{\{\alpha\}}(\mathfrak{A}, \mathfrak{C})$ and $D_{i} \in \mathfrak{D}_{\{\alpha\}}(\mathscr{S})$.

Consequently, we have $\mathscr{S}^{*} \in \mathfrak{W}_{\{\alpha\}}(\mathfrak{A}, \mathfrak{C})$. Next, we show that for any subspace $\mathcal{W}$ such that it is $\left(A_{i}+D_{i} C_{i}\right)$-invariant $\left(i \in \Phi_{\alpha}\right)$ and is contained in $\bigcap_{j \in \Phi_{\alpha}}\left(\mathcal{S}_{\alpha}+\operatorname{Ker} C_{j}\right) \bigcap \mathcal{K}_{\alpha}$, we have $\mathcal{W} \subseteq \mathscr{S}^{*}$. If $\mathcal{W} \subseteq \mathcal{S}_{\alpha}$, then it follows that $\mathcal{W} \subseteq \mathscr{S}^{*}$, since $\mathcal{S}_{\alpha} \subseteq \mathscr{S}^{*}$. Therefore, we consider the case where $\mathcal{S}_{\alpha} \subset$ $\mathcal{W}$. We have, $A_{i}^{-1} \mathcal{W} \cap \operatorname{Ker} C_{i}=\left(A_{i}+D_{i} C_{i}\right)^{-1} \mathcal{W} \cap \operatorname{Ker} C_{i}$, and consequently

$$
\begin{aligned}
\bigcap_{i \in \Phi_{\alpha}} & \left(\mathcal{S}_{\alpha}+A_{i}^{-1} \mathcal{W} \cap \operatorname{Ker} C_{i}\right) \\
& =\bigcap_{i \in \Phi_{\alpha}}\left(\mathcal{S}_{\alpha}+\left(A_{i}+D_{i} C_{i}\right)^{-1} \mathcal{W} \cap \operatorname{Ker} C_{i}\right)
\end{aligned}
$$

It follows that $\mathcal{W} \subseteq \mathscr{S}^{0}$. If $\mathcal{W} \subseteq \mathscr{S}^{\mu-1}$, then

$$
\begin{aligned}
\mathscr{S}^{\mu} & =\bigcap_{i \in \Phi_{\alpha}}\left(\mathcal{S}_{\alpha}+\left(A_{i}^{-1} \mathscr{S}^{\mu-1} \cap \operatorname{Ker} C_{i}\right)\right) \bigcap \mathcal{K}_{\alpha} \\
& \supseteq \bigcap_{i \in \Phi_{\alpha}}\left(\mathcal{S}_{\alpha}+\left(A_{i}^{-1} \mathcal{W} \cap \operatorname{Ker} C_{i}\right)\right) \bigcap \mathcal{K}_{\alpha} \\
& \left.=\bigcap_{i \in \Phi_{\alpha}}\left(\mathcal{S}_{\alpha}+\left(A_{i}+D_{i} C_{i}\right)^{-1} \mathcal{W} \cap \operatorname{Ker} C_{i}\right)\right) \bigcap \mathcal{K}_{\alpha} \\
& \supseteq \bigcap_{i \in \Phi_{\alpha}}\left(\mathcal{S}_{\alpha}+\left(\mathcal{W} \cap \operatorname{Ker} C_{i}\right)\right) \bigcap \mathcal{K}_{\alpha} \\
& =\bigcap_{i \in \Phi_{\alpha}}\left(\mathcal{W} \cap\left(\mathcal{S}_{\alpha}+\operatorname{Ker} C_{i}\right)\right) \bigcap \mathcal{K}_{\alpha}=\mathcal{W}
\end{aligned}
$$

where we used the fact that $\mathcal{W} \subseteq\left(A_{i}+D_{i} C_{i}\right)^{-1} \mathcal{W}$, the modular distributive rule [28] (if $\mathcal{S}_{\alpha} \subset \mathcal{W}$, then $\mathcal{S}_{\alpha}+\left(\mathcal{W} \cap \operatorname{Ker} C_{i}\right)=$ $\left.\mathcal{W} \cap\left(\mathcal{S}_{\alpha}+\operatorname{Ker} C_{i}\right)\right)$, and $\mathcal{W} \subset \bigcap_{j \in \Phi_{\alpha}}\left(\mathcal{S}_{\alpha}+\operatorname{Ker} C_{j}\right) \bigcap \mathcal{K}_{\alpha}$. Consequently, $\mathcal{W} \subseteq \mathscr{S}^{\mu}$; and hence $\mathcal{W} \subseteq \mathscr{S}^{*}$. This shows that $\mathscr{S}^{*}$ is the largest $\left(A_{i}+D_{i} C_{i}\right)$-invariant $\left(i \in \Phi_{\alpha}\right)$ which is contained in $\bigcap_{i \in \Phi_{\alpha}}\left(\mathcal{S}_{\alpha}+\operatorname{Ker} C_{i}\right) \bigcap \mathcal{K}_{\alpha}$.

We are now in the position to introduce our proposed alternative characterization of an unobservability subspace for system $(\mathfrak{A}, \mathfrak{C}, \Pi)$.

Theorem 4.5: Let $\mathcal{S}_{\alpha} \subseteq \mathcal{K}_{\alpha}$ and consider the family $\mathcal{G}_{(\mathfrak{A}, \mathfrak{C})}^{\alpha}=\left\{\mathscr{S} \mid \mathscr{S}=\bigcap_{i \in \Phi_{\alpha}}\left(\mathcal{S}_{\alpha}+\left(A_{i}^{-1} \mathscr{S} \cap \operatorname{Ker} C_{i}\right)\right) \bigcap \mathcal{K}_{\alpha}\right\}$. Then $\mathcal{S}_{\alpha} \in \mathfrak{S}_{\alpha}(\mathfrak{A}, \mathfrak{C})$ if and only if $\mathcal{S}_{\alpha} \in \mathfrak{W}_{\{\alpha\}}(\mathfrak{A}, \mathfrak{C})\left(\mathcal{S}_{\alpha}\right.$ is conditioned invariant for class $\left.\Phi_{\alpha}\right)$ and $\mathcal{S}_{\alpha}=\mathscr{S}^{*}$, where $\mathscr{S}^{*}$ is the maximal element of $\mathcal{G}_{(\mathfrak{A}, \mathfrak{C})}^{\alpha}$.

Proof: (If part) If $\mathcal{S}_{\alpha} \in \mathfrak{W}_{\{\alpha\}}(\mathfrak{A}, \mathfrak{C})$ and $\mathcal{S}_{\alpha}=\mathscr{S}^{*}$ hold, then according to Lemma 4.4, it follows that $\mathcal{S}_{\alpha}=<<\bigcap_{j \in \Phi_{\alpha}}\left(\mathcal{S}_{\alpha}+\right.$ $\left.\operatorname{Ker} C_{j}\right) \bigcap \mathcal{K}_{\alpha} \mid A_{i}+D_{i} C_{i}>>_{i \in \Phi_{\alpha}}$, and hence using Theorem 4.2, one gets $\mathcal{S}_{\alpha} \in \mathfrak{S}_{\alpha}(\mathfrak{A}, \mathfrak{C})$.

(Only if part) If $\mathcal{S}_{\alpha}$ is an unobservability subspace for class $\Phi_{\alpha}$, it follows that $\mathcal{S}_{\alpha} \in \mathfrak{W}_{\{\alpha\}}(\mathfrak{A}, \mathfrak{C})$, and according to Lemma 4.4 one gets $\mathcal{S}_{\alpha}=\mathscr{S}^{*}$.

The above theorem provides a characteristic for the u.o.s. of the MJLS's that is similar to what Theorem 2.1 provides for u.o.s. of a system having only a single mode. Let $\mathcal{L} \subset \mathcal{K}_{\alpha}$ be an arbitrary subspace and let us denote $\mathfrak{S}_{\alpha}(\mathfrak{A}, \mathfrak{C}, \mathcal{L})$ as the family of u.o.s. for a class $\Phi_{\alpha}$ of $(\mathfrak{A}, \mathfrak{C}, \Pi)$ that contains $\mathcal{L}$. It can be shown that $\mathfrak{S}_{\alpha}(\mathfrak{A}, \mathfrak{C}, \mathcal{L})$ is closed under the operation of subspace intersection. The next lemma provides the necessary and sufficient conditions for $\mathfrak{S}_{\alpha}(\mathfrak{A}, \mathfrak{C}, \mathcal{L})$ to be nonempty.

Lemma 4.6: The family of subspaces $\mathfrak{S}_{\alpha}(\mathfrak{A}, \mathfrak{C}, \mathcal{L})$ is nonempty if and only if the family of conditioned invariant subspaces for $\Phi_{\alpha}$ in $\mathcal{K}_{\alpha}$ that contains $\mathcal{L}$ is nonempty, i.e. there exists a subspace $\mathcal{W}_{\alpha}$ such that $\mathcal{W}_{\alpha} \in \mathfrak{W}_{\{\alpha\}}(\mathfrak{A}, \mathfrak{C}, \mathcal{L})$ and $\mathcal{W}_{\alpha} \subseteq \mathcal{K}_{\alpha}$.

Proof: (If part) If there exists an $\mathcal{W}_{\alpha}$ such that $\mathcal{W}_{\alpha} \in$ $\mathfrak{W}_{\{\alpha\}}(\mathfrak{A}, \mathfrak{C}, \mathcal{L})$, then there exist $D_{i} \in \mathfrak{D}_{\{\alpha\}}\left(\mathcal{W}_{\alpha}\right), i \in \Phi_{\alpha}$ such that $\mathcal{W}_{\alpha}$ is $\left(A_{i}+D_{i} C_{i}\right)$-invariant. Define $H_{j}=0, j \in \Phi_{\alpha}$. It follows that $\bigcap_{j \in \Phi_{\alpha}} \operatorname{Ker} H_{j} C_{j} \cap \mathcal{K}_{\alpha}=\mathcal{K}_{\alpha}$. Let us define $\mathcal{S}_{\alpha}$ 
as the largest element of a class of $\left(A_{i}+D_{i} C_{i}\right)$-invariant subspaces $\left(i \in \Phi_{\alpha}\right)$ that is contained in $\mathcal{K}_{\alpha}$ (denoted by $\mathfrak{V}_{\alpha}\left(\mathcal{K}_{\alpha}\right)$ ), i.e. $\mathcal{S}_{\alpha}=<<\mathcal{K}_{\alpha} \mid A_{i}+D_{i} C_{i}>>_{i \in \Phi_{\alpha}}$. It follows that $\mathfrak{V}_{\alpha}\left(\mathcal{K}_{\alpha}\right)$ is nonempty since $\mathcal{W}_{\alpha} \in \mathfrak{V}_{\alpha}\left(\mathcal{K}_{\alpha}\right)$. Moreover, $\mathfrak{V}_{\alpha}\left(\mathcal{K}_{\alpha}\right)$ is closed under the operation of subspace addition, and hence it has a supremal element which is $\mathcal{S}_{\alpha}$. Moreover, we have $\mathcal{L} \subseteq \mathcal{S}_{\alpha}$, since $\mathcal{L} \subseteq \mathcal{W}_{\alpha}$ and $\mathcal{W}_{\alpha} \subseteq \mathcal{S}_{\alpha}$. Therefore, $\mathcal{S}_{\alpha} \in \mathfrak{S}_{\alpha}(\mathfrak{A}, \mathfrak{C}, \mathcal{L})$.

(Only if part) If there exists an $\mathcal{S}_{\alpha} \in \mathfrak{S}_{\alpha}(\mathfrak{A}, \mathfrak{C}, \mathcal{L})$, then it is clear that $\mathcal{S}_{\alpha} \in \mathfrak{W}_{\{\alpha\}}(\mathfrak{A}, \mathfrak{C}, \mathcal{L})$ and $\mathcal{S}_{\alpha} \subseteq \mathcal{K}_{\alpha}$.

Consequently, if the conditions of Lemma 4.6 are satisfied then $\mathfrak{S}_{\alpha}(\mathfrak{A}, \mathfrak{C}, \mathcal{L})$ has as an infimal element (denoted by $\mathcal{S}_{\alpha}^{*}$ ). This property is crucial for application of the u.o.s. to the FDI problem of the MJLS's. The next algorithm provides a procedure for constructing $\mathcal{S}_{\alpha}^{*}$.

Algorithm 3: Let $\mathcal{W}_{\alpha}^{*}=\inf \mathfrak{W}_{\{\alpha\}}(\mathfrak{A}, \mathfrak{C}, \mathcal{L})$ and conditions of Lemma 4.6 be satisfied. Define the sequence $\mathcal{Z}^{\mu}$ according to

$$
\mathcal{Z}^{0}=\mathcal{X} ; \quad \mathcal{Z}^{\mu}=\bigcap_{i \in \Phi_{\alpha}}\left(\mathcal{W}_{\alpha}^{*}+\left(A_{i}^{-1} \mathcal{Z}^{\mu-1} \cap \operatorname{Ker} C_{i}\right)\right) \bigcap \mathcal{K}_{\alpha}
$$

Consequently, $\mathcal{S}_{\alpha}^{*}=\mathcal{Z}^{\mu}$, whenever $\mathcal{Z}^{\mu+1}=\mathcal{Z}^{\mu}$.

To analyze the above algorithm note that the sequence $\mathcal{Z}^{\mu}$ is non-increasing and $\mathcal{Z}^{k+1}=\mathcal{Z}^{k}$ for $k \geq n-\operatorname{dim}\left(\mathcal{W}_{\alpha}^{*}\right)$. Let $\mathcal{Z}^{*}=\mathcal{Z}^{k}$. According to Lemma 4.4 and the fact that $\mathcal{W}_{\alpha}^{*} \subseteq \mathcal{K}_{\alpha}$ (Lemma 4.6), $\mathcal{Z}^{*}=<<\bigcap_{j \in \Phi_{\alpha}}\left(\mathcal{W}_{\alpha}^{*}+\right.$ $\left.\operatorname{Ker} C_{j}\right) \cap \mathcal{K}_{\alpha} \mid A_{i}+D_{i} C_{i}>>_{i \in \Phi_{\alpha}}$ for some $D_{i} \in \mathfrak{D}_{\{\alpha\}}\left(\mathcal{W}_{\alpha}^{*}\right)$. Following along the same lines as in Lemma 4.1, one can obtain the maps $H_{j}$ 's such that $\mathcal{W}_{\alpha}^{*}+\operatorname{Ker} C_{j}=\operatorname{Ker} H_{j} C_{j}$ and $\mathcal{Z}^{*}=<<\bigcap_{j \in \Phi_{\alpha}} \operatorname{Ker} H_{j} C_{j} \bigcap \mathcal{K}_{\alpha} \mid A_{i}+D_{i} C_{i}>>_{i \in \Phi_{\alpha}}$, therefore $\mathcal{Z}^{*}$ is an u.o.s. according to Definition 8. Moreover, it follows that $\mathcal{L} \subseteq \mathcal{W}_{\alpha}^{*} \subseteq \mathcal{Z}^{*}\left(\mathcal{Z}^{*} \in \mathfrak{W}_{\{\alpha\}}(\mathfrak{A}, \mathfrak{C}, \mathcal{L})\right.$ and $\mathcal{W}_{\alpha}^{*}=\inf \mathfrak{W}_{\{\alpha\}}(\mathfrak{A}, \mathfrak{C}, \mathcal{L})$ ); hence $\mathcal{Z}^{*} \in \mathfrak{S}_{\alpha}(\mathfrak{A}, \mathfrak{C}, \mathcal{L})$, and consequently $\mathcal{S}_{\alpha}^{*} \subseteq \mathcal{Z}^{*}$.

On the other hand, according to Theorem 4.5 we have $\mathcal{S}_{\alpha}^{*}=$ $\mathcal{S}^{n}$, where $\mathcal{S}^{0}=\mathcal{X}$ and $\mathcal{S}^{\mu}=\bigcap_{i \in \Phi_{\alpha}}\left(\mathcal{S}_{\alpha}^{*}+\left(A_{i}^{-1} \mathcal{S}^{\mu-1} \cap\right.\right.$ $\left.\left.\operatorname{Ker} C_{i}\right)\right) \cap \mathcal{K}_{\alpha}, \mu \in \mathbf{n}$. Since $\mathcal{W}_{\alpha}^{*} \subseteq \mathcal{S}_{\alpha}^{*}$, it can be shown by induction that $\mathcal{Z}^{\mu} \subseteq \mathcal{S}^{\mu}, \mu \in \mathbf{n}$. Indeed, $\mathcal{Z}^{0}=\mathcal{S}^{0}$, and if $\mathcal{Z}^{\mu-1} \subseteq \mathcal{S}^{\mu-1}$, then

$$
\begin{aligned}
\mathcal{Z}^{\mu} & =\bigcap_{i \in \Phi_{\alpha}}\left(\mathcal{W}_{\alpha}^{*}+\left(A_{i}^{-1} \mathcal{Z}^{\mu-1} \cap \operatorname{Ker} C_{i}\right)\right) \bigcap \mathcal{K}_{\alpha} \\
& \subseteq \bigcap_{i \in \Phi_{\alpha}}\left(\mathcal{S}_{\alpha}^{*}+\left(A_{i}^{-1} \mathcal{S}^{\mu-1} \cap \operatorname{Ker} C_{i}\right)\right) \bigcap \mathcal{K}_{\alpha}=\mathcal{S}^{\mu}
\end{aligned}
$$

Consequently, $\mathcal{Z}^{*} \subseteq \mathcal{S}_{\alpha}^{*}$.

It follows from the above algorithm and Lemma 4.4 that $\mathfrak{D}_{\{\alpha\}}\left(\mathcal{W}_{\alpha}^{*}\right) \subset \mathfrak{D}_{\alpha}\left(\mathcal{S}_{\alpha}^{*}\right)$. Therefore, the maps $D_{i}$ 's for $\mathcal{S}_{\alpha}^{*}$ can be found from $\mathcal{W}_{\alpha}^{*}$ and once $\mathcal{S}_{\alpha}^{*}$ is found from Algorithm 3, the maps $H_{i}$ 's can also be computed from $\mathcal{S}_{\alpha}^{*}$ and the expression $\mathcal{S}_{\alpha}^{*}+\operatorname{Ker} C_{j}=\operatorname{Ker} H_{j} C_{j}$.

Consequently, for a closed class $\Phi_{\alpha}, \alpha \leq \delta$, we have $\mathcal{K}_{\alpha}=\mathcal{X}$, and hence the conditions of Lemma 4.6 are satisfied. However, for an open class $\Phi_{\alpha}, \alpha>\delta$, we have $\mathcal{K}_{\alpha}=\bigcap_{l \in \mathrm{N}_{\alpha}^{d}} \mathcal{S}_{l}=\bigcap_{l \in \mathrm{N}_{\alpha}} \mathcal{S}_{l}$. Hence, the unobservability subspaces $\mathcal{S}_{l}$ for all classes $\Phi_{l}, l \in \mathrm{N}_{\alpha}$ that are accessible from the class $\Phi_{\alpha}$ should be constructed in such a way that the conditions of Lemma 4.6 are satisfied. In other words, for an open class $\Phi_{\alpha}$, the $\left|\mathrm{N}_{\alpha}\right|+1$ subspaces should be constructed in such a way that the conditions of Lemma 4.6 are satisfied. The following algorithm provides a procedure for constructing $\left|\mathrm{N}_{\alpha}\right|+1$ subspaces for an open class $\Phi_{\alpha}$.

Algorithm 4: Step 1: First, construct the unobservability subspaces for the closed classes in $\mathrm{N}_{\alpha}^{c}$ as follows. For each class $\Phi_{\alpha_{i}^{c}}, \alpha_{i}^{c} \in \mathrm{N}_{\alpha}^{c}$, the sequence of subspaces are generated as follows

$$
\mathcal{Z}^{\mu}=\inf \mathfrak{S}_{\alpha_{i}^{c}}\left(\mathfrak{A}, \mathfrak{C}, \mathcal{Z}^{\mu-1}\right), \quad \mathcal{Z}^{\mu+1}=\inf \mathfrak{W}_{\Gamma_{\alpha_{i}^{c}}^{\alpha^{*}}}\left(\mathfrak{A}, \mathfrak{C}, \mathcal{Z}^{\mu}\right)
$$

where $\mathcal{Z}^{0}=\mathcal{L}$ and the subspaces $\inf \mathfrak{S}_{\alpha_{i}^{c}}\left(\mathfrak{A}, \mathfrak{C}, \mathcal{Z}^{\mu-1}\right)$ and $\inf \mathfrak{W}_{\Gamma_{\alpha_{i}^{*}}^{\alpha_{c}^{*}}}\left(\mathfrak{A}, \mathfrak{C}, \mathcal{Z}^{\mu}\right)$ can be found from Algorithms 3 and 2, respectively. Whenever $\mathcal{Z}^{\mu}=\mathcal{Z}^{\mu+1}$, then $\mathcal{S}_{\alpha_{i}^{c}}=\mathcal{Z}^{\mu}$. Due to the fact that $\Phi_{\alpha_{i}^{c}}$ is closed, the subspace $\mathcal{K}_{\alpha_{i}^{c}}$ in Algorithm 3 is the same as $\mathcal{X}$.

Step 2: Second, construct the unobservability subspaces for open classes in $\mathrm{N}_{\alpha}^{o}$ one by one from the first open class $\alpha_{1}^{o}$ as follows. For each open class $\alpha_{i}^{o}$, the sequence of subspaces are generated as follows

$$
\mathcal{Z}^{\mu}=\inf \mathfrak{S}_{\alpha_{i}^{o}}\left(\mathfrak{A}, \mathfrak{C}, \mathcal{Z}^{\mu-1}\right), \quad \mathcal{Z}^{\mu+1}=\inf \mathfrak{W}_{\Gamma_{\alpha_{i}^{\alpha}}^{\alpha^{*}}}\left(\mathfrak{A}, \mathfrak{C}, \mathcal{Z}^{\mu}\right)
$$

with $\mathcal{Z}^{0}=\mathcal{L}$. Whenever $\mathcal{Z}^{\mu}=\mathcal{Z}^{\mu+1}$, then $\mathcal{S}_{\alpha_{i}^{o}}=\mathcal{Z}^{\mu}$. For obtaining the subspace inf $\mathfrak{S}_{\alpha_{i}^{o}}\left(\mathfrak{A}, \mathfrak{C}, \mathcal{Z}^{\mu-1}\right)$, the subspace $\mathcal{K}_{\alpha_{i}^{o}}=\bigcap_{l \in \mathrm{N}_{\alpha_{i}^{o}}} \mathcal{S}_{l}$ can be found easily from the subspaces that are constructed in Step 1 and the subspaces that are obtained for classes $\Phi_{\alpha_{i}^{o}}, j<i$.

Step 3: Finally, $\mathcal{S}_{\alpha}^{*}$ is found from Algorithm 3 by setting $\mathcal{K}_{\alpha}=\bigcap_{l \in \mathrm{N}_{\alpha}^{d}} \mathcal{S}_{l}$.

To analyze the above algorithm note that every subspace of the sequence in Step 1 contains the previous one (i.e. $\mathcal{Z}^{\mu-1} \subseteq$ $\mathcal{Z}^{\mu}$ ). Whenever $\mathcal{Z}^{\mu}=\mathcal{Z}^{\mu+1}$, it follows that $\mathcal{S}_{\alpha_{i}^{c}}=\mathcal{Z}^{\mu} \in$ $\mathfrak{S}_{\alpha_{i}^{c}}(\mathfrak{A}, \mathfrak{C}, \mathcal{L})$ and $\mathcal{S}_{\alpha_{i}^{c}}=\mathcal{Z}^{\mu} \in \mathfrak{W}_{\Gamma_{\alpha c}^{\alpha^{*}}}(\mathfrak{A}, \mathfrak{C}, \mathcal{L})$. Hence, $\mathcal{S}_{\alpha_{i}^{c}}$ is both u.o.s. for class $\Phi_{\alpha_{i}^{c}}$ and conditioned invariant for all classes in $\Gamma_{\alpha_{i}^{c}}^{\alpha^{*}}$. In Step 2, since the unobservability subspaces are found sequentially, it follows that for each open class $\Phi_{\alpha_{i}^{o}}$, the subspace $\mathcal{K}_{\alpha_{i}^{o}}=\bigcap_{l \in \mathrm{N}_{\alpha_{i}^{o}}^{d}} \mathcal{S}_{l}=\bigcap_{l \in \mathrm{N}_{\alpha_{i}^{o}}^{d}, \alpha_{i}^{o} \in \Gamma_{l}^{\alpha^{*}}} \mathcal{S}_{l} \in$ $\mathfrak{W}_{\left\{\alpha_{i}^{\circ}\right\}}(\mathfrak{A}, \mathfrak{C}, \mathcal{L})$ (the class $\mathfrak{W}_{\left\{\alpha_{i}^{\circ}\right\}}(\mathfrak{A}, \mathfrak{C}, \mathcal{L})$ is closed under intersection operation). This is due to the fact that for all classes $l \in \mathrm{N}_{\alpha_{i}^{o}}^{d}$ either $\alpha_{i}^{o} \in \Gamma_{l}^{\alpha^{*}}$ or there exists $k \in \mathrm{N}_{\alpha_{i}^{o}}^{d}$ such that $l \in \mathrm{N}_{k}$ and $\alpha_{i}^{o} \in \Gamma_{k}^{\alpha^{*}}$.

For the first scenario, according to Steps 1 and 2, we have $\mathcal{S}_{l} \in \mathfrak{W}_{\left\{\alpha_{i}^{\circ}\right\}}(\mathfrak{A}, \mathfrak{C}, \mathcal{L})$. For the second scenario, we have $\mathcal{S}_{k} \subset$ $\mathcal{S}_{l}$ and $\mathcal{S}_{k} \in \mathfrak{W}_{\left\{\alpha_{i}^{o}\right\}}(\mathfrak{A}, \mathfrak{C}, \mathcal{L})$. This shows that it is not required to construct all $\mathcal{S}_{l}, l \in \mathrm{N}_{\alpha_{i}^{o}}^{d}$ in a manner that they belong to $\mathfrak{W}_{\left\{\alpha_{i}^{o}\right\}}(\mathfrak{A}, \mathfrak{C}, \mathcal{L})$. In other words, only u.o.s. of classes $l \in$ $\mathrm{N}_{\alpha_{i}^{o}}^{d}, \alpha_{i}^{o} \in \Gamma_{l}^{\alpha^{*}}$ are required to belong to $\mathfrak{W}_{\left\{\alpha_{i}^{o}\right\}}(\mathfrak{A}, \mathfrak{C}, \mathcal{L})$. Hence, the necessary and sufficient conditions of Lemma 4.6 are satisfied and $\mathcal{S}_{\alpha_{i}^{o}}$ can be found by invoking Algorithm 3 . Similarly, in Step 3 it is clear that $\mathcal{K}_{\alpha}$ satisfies the conditions of Lemma 4.6 and $\mathcal{S}_{\alpha}^{*}$ can be easily determined.

Remark 2: As shown subsequently in Section V, for an open class the unobservability subspace $\mathcal{S}_{l}^{*}$ is required to contain the other given subspace (here denoted by $\mathcal{L}_{l}$ ) which represents fault signatures in the $l$-th operational mode of the MJLS. In this case, Algorithm 4 can be invoked by setting $\mathcal{Z}^{0}=\mathcal{L}_{l}+\mathcal{L}$. 
As discussed above, for an open class $\Phi_{\alpha}$, one needs to find $\left|\mathrm{N}_{\alpha}\right|+1$ unobservability subspaces $\mathcal{S}_{\alpha}$ and $\mathcal{S}_{l}, l \in \mathrm{N}_{\alpha}$. Moreover, we have $\mathcal{S}_{\alpha} \subseteq \mathcal{S}_{l}$. Let us define $P_{\alpha}$ and $P_{l}, l \in$ $\mathrm{N}_{\alpha}$ as the canonical projections of $\mathcal{X}$ on $\mathcal{X} / \mathcal{S}_{\alpha}$ and $\mathcal{X} / \mathcal{S}_{l}$, respectively. Moreover, for each class $l \in \mathrm{N}_{\alpha}$ and a class $k \in \Gamma_{l}^{\alpha}$, we have $\mathcal{S}_{k} \subseteq \mathcal{S}_{l}$. We need to define the canonical projection $T_{l, k}$ of $\mathcal{X} / \mathcal{S}_{k}$ on $\mathcal{X} / \mathcal{S}_{l}$. Note that $P_{l}=T_{l, k} P_{k}$. Consider the following MJLS

$$
\begin{aligned}
\dot{x}(t) & =\left(A_{\lambda(t)}+D_{\lambda(t)} C_{\lambda(t)}\right) x(t) \\
\tilde{y}(t) & =H_{\lambda(t)} C_{\lambda(t)} x(t)
\end{aligned}
$$

where $x(0)=x_{0}, \lambda(0)=i_{0}$, and the maps $D_{i}, H_{i}$ are found such that $\mathcal{S}_{\alpha}=<<\bigcap_{k \in \Phi_{\alpha}} \operatorname{Ker} H_{k} C_{k} \bigcap \mathcal{K}_{\alpha} \mid A_{i}+$ $D_{i} C_{i}>>_{i \in \Phi_{\alpha}}$ and $\mathcal{S}_{l}=<<\bigcap_{k \in \Phi_{l}} \operatorname{Ker} H_{k} C_{k} \bigcap \mathcal{K}_{l} \mid A_{i}+$ $D_{i} C_{i}>>_{i \in \Phi_{l}}, l \in \mathrm{N}_{d}$.

We are interested in investigating the convergence properties of the trajectories of the factor system of (4) on subspace $\mathcal{X} / \mathcal{S}_{\alpha}$ when $\lambda(t) \in \Phi_{\alpha}$, and on subspaces $\mathcal{X} / \mathcal{S}_{l}$ when $\lambda(t) \in$ $\Phi_{l}, l \in \mathrm{N}_{\alpha}$. It is assumed that the initial mode $i_{0}$ belongs to an open class $\Phi_{\alpha}$. Let us define $\bar{x}_{\alpha}(t)=P_{\alpha} x(t)$ for $\lambda(t) \in \Phi_{\alpha}$. Whenever $\lambda(t)$ leaves the class $\Phi_{\alpha}$ at time $t=\nu$ and enters $\Phi_{l}, l \in \mathrm{N}_{\alpha}$ we define the factor state $\bar{x}_{l}(t)=P_{l} x(t)$ for all $t$ such that $\lambda(t) \in \Phi_{l}$. Consequently, we have $\bar{x}_{l}(\nu)=$ $T_{l, \alpha} \bar{x}_{\alpha}\left(\nu^{-}\right)$and the dimension of $x_{l}$ is not greater than that of $x_{\alpha}$. Moreover, for each $i \in \Phi_{\alpha}$ and $j \in \Phi_{l}$, one can determine the induced maps $\bar{A}_{i}=\left(A_{i}+D_{i} C_{i}: \mathcal{X} / \mathcal{S}_{\alpha}\right)$ and $\bar{A}_{j}=$ $\left(A_{j}+D_{j} C_{j}: \mathcal{X} / \mathcal{S}_{l}\right)$, where we have $P_{\alpha}\left(A_{i}+D_{i} C_{i}\right)=\bar{A}_{i} P_{\alpha}$ and $P_{l}\left(A_{j}+D_{i} C_{j}\right)=\bar{A}_{i} P_{l}$. Let $M_{i}, i \in \Phi_{\alpha}$ and $M_{j}, j \in \Phi_{l}$ be unique solutions to $M_{i} P_{\alpha}=H_{i} C_{i}$ and $M_{j} P_{l}=H_{j} C_{j}$, respectively.

Let us define $\left\{\nu_{k}, k=0,1, \ldots, \varrho\right\}$ as the jump instants of $\bar{\lambda}(t)$ satisfying $0=\nu_{0}<\nu_{1}<\nu_{2}<\cdots<\nu_{\varrho}$. It follows that when $i_{0} \in \Phi_{\alpha}$, the maximum number of jumps $\varrho$ between the classes is $\left|\mathrm{N}_{\alpha}\right|$. This corresponds to the case when there exists only one closed class in $\mathrm{N}_{\alpha}$ (i.e. $\mathrm{N}_{\alpha}^{c}=\left\{\alpha_{1}^{c}\right\}$ ), and for the open classes we have $\mathrm{N}_{\alpha}^{o}=\left\{\alpha_{1}^{o}, \ldots, \alpha_{\left|\mathrm{N}_{\alpha}\right|-1}^{o}\right\}$, where $\alpha_{j}^{o} \in \mathrm{N}_{\alpha_{j+1}^{o}}$, $j=1, \ldots,\left|\mathrm{N}_{\alpha}\right|-2$, and $\alpha_{1}^{c} \in \mathrm{N}_{\alpha_{1}^{o}}$. In other words, there exists a nonzero probability for a sequence of jumps that has started from class $\Phi_{\alpha}$ to $\Phi_{\alpha_{\left|N_{\alpha}\right|-1}^{o}}$, to be followed by jumps from $\Phi_{\alpha_{j}^{o}}$ to $\Phi_{\alpha_{j-1}^{o}}, j=\left|\mathrm{N}_{\alpha}\right|-1, \ldots, 2$, and finally the last jump taking place from class $\Phi_{\alpha_{1}^{o}}$ to $\Phi_{\alpha_{1}^{c}}$. The factor system of (4) on the subspaces $\mathcal{X} / \mathcal{S}_{\alpha}$ and $\mathcal{X} / \mathcal{S}_{l}$ can be written as

$$
\begin{aligned}
\dot{\bar{x}}_{\bar{\lambda}(t)}(t) & =\bar{A}_{\lambda(t)} \bar{x}_{\bar{\lambda}(t)}(t), \quad t \neq \nu_{k}, \quad \lambda(t) \in \Phi_{\bar{\lambda}(t)} \\
\bar{x}_{\bar{\lambda}\left(\nu_{k}\right)}\left(\nu_{k}\right) & =T_{\bar{\lambda}\left(\nu_{k}\right), \bar{\lambda}\left(\nu_{k}^{-}\right)} \bar{x}_{\bar{\lambda}\left(\nu_{k}^{-}\right)}\left(\nu_{k}^{-}\right), \quad t=\nu_{k},
\end{aligned}
$$

with measurement $\tilde{y}(t)=M_{\lambda(t)} \bar{x}_{\bar{\lambda}(t)}(t)$.

We now would like to investigate the stochastic convergence of system (5) with an initial mode $i_{0}$, i.e. to determine whether there exists a finite positive constant $T\left(\bar{x}_{0}\right)$ such that for any initial condition $\bar{x}_{0}$, we have $\mathbb{E}\left[\int_{0}^{\infty}\left\|\bar{x}_{\bar{\lambda}(t)}(t)\right\|^{2} d t \mid \bar{x}_{0}, i_{0}\right] \leq$ $T\left(\bar{x}_{0}\right)$. Due to the fact that the number of jumps among the classes are finite, one can guarantee the stochastic convergence of system (5) by ensuring the stochastic convergence of each class of operations in (5) individually, i.e. for each $\bar{\lambda}(t) \in$ $\mathrm{N}_{\alpha} \cup \alpha$.

Lemma 4.7: System (5) is stochastically convergent with an initial mode $i_{0} \in \Phi_{\alpha}$ if the following systems are all stochastically convergent, namely

$$
\dot{\bar{x}}_{j}(t)=\bar{A}(\lambda(t)) \bar{x}_{j}(t), \lambda(t) \in \Phi_{j}, \forall j \in \mathrm{N}_{\alpha} \cup \alpha
$$

Proof: Let us assume that the maximum number of jumps among the classes is denoted by $\varrho$ where the jump instants are specified by $\nu_{k}, k=1, \ldots, \varrho$. Therefore, system starts from an initial mode $i_{0}$ and then jumps to the next class $\Phi_{l}, l \in \mathrm{N}_{\alpha}$ at $t=\nu_{1}$. Due to the fact that each class is stochastically convergent there exists a constant $T\left(\bar{x}_{0}, i_{0}\right)$ such that $\mathbb{E}\left[\int_{0}^{\nu_{1}}\left\|\bar{x}_{\alpha}(t)\right\|^{2} d t \mid \bar{x}_{0}, i_{0}\right] \leq T\left(\bar{x}_{0}, i_{0}\right)$. Moreover, the initial condition corresponding to the trajectory of the second class is set as $\bar{x}_{l}\left(\nu_{1}\right)=T_{l, \alpha} \bar{x}_{\alpha}\left(\nu_{1}^{-}\right)$. Similarly, due to the convergence of the second class we have $\mathbb{E}\left[\int_{\nu_{1}}^{\nu_{2}}\left\|\bar{x}_{l}(t)\right\|^{2} d t\right] \leq$ $T\left(\bar{x}\left(\nu_{1}\right), i_{1}\right)$, where $i_{1}=\lambda\left(\nu_{1}\right)$. Similar arguments can be made for all the other classes. Consequently, we have $\mathbb{E}\left[\int_{0}^{\infty}\left\|\bar{x}_{\bar{\lambda}(t)}(t)\right\|^{2} d t\right] \leq \sum_{j=0}^{\varrho} T\left(\bar{x}\left(\nu_{j}\right), i_{j}\right)$, which shows that system (5) is stochastically convergent.

Remark 3: It is known that if system (6) is stochastically convergent then it is also mean-square (MS)-stable, i.e. $\lim _{t \rightarrow \infty}\left\|\bar{x}_{j}(t)\right\|^{2}=0$. Therefore, it can be concluded that if the conditions of Lemma 4.7 are satisfied then $\lim _{t \rightarrow \infty}\left\|x_{\bar{\lambda}(t)}(t)\right\|^{2}=0$, and system (5) is MS-stable.

It should be noted that if the initial condition $i_{0}$ belongs to the closed class $\Phi_{\alpha}$, we will have $\lambda(t)=\alpha, t>0$, and the dimension of the factor system (5) is constant. Our last result in this section is concerned with MS-detectability of the MJLS (5).

Definition 9 ([22]): System (5) is said to be MSdetectable when there exist maps $G_{i}, i \in \Phi_{k}, k \in \mathrm{N}_{\alpha} \cup \alpha$ for which the following system

$$
\begin{gathered}
\dot{\bar{x}}_{\bar{\lambda}(t)}(t)=\left(\bar{A}_{\lambda(t)}+G_{\lambda(t)} M_{\lambda(t)}\right) \bar{x}_{\bar{\lambda}(t)}(t), t \neq \nu_{k}, \lambda(t) \in \Phi_{\bar{\lambda}(t)} \\
\bar{x}_{\bar{\lambda}\left(\nu_{k}\right)}\left(\nu_{k}\right)=T_{\bar{\lambda}\left(\nu_{k}\right), \bar{\lambda}_{\left(\nu_{k}^{-}\right)}} \bar{x}_{\bar{\lambda}\left(\nu_{k}^{-}\right)}\left(\nu_{k}^{-}\right), \quad t=\nu_{k}
\end{gathered}
$$

is MS-stable. Moreover if system (5) is MS-detectable, the subspaces $\mathcal{S}_{\alpha}$ and $\mathcal{S}_{l}, l \in \mathrm{N}_{\alpha}$ are said to be outer MSdetectable.

According to Lemma 4.7, system (5) is MS-detectable if there exist $X_{i}>0$ and $L_{i}, i \in \Phi_{k}, k \in \mathrm{N}_{\alpha} \cup \alpha$ having appropriate dimensions such that [22]

$$
\bar{A}_{i}^{\prime} X_{i}+X_{i} \bar{A}_{i}+M_{i}^{\prime} L_{i}^{\prime}+L_{i} M_{i}+\sum_{j \in \Phi_{k}} \pi_{i j} X_{j}<0
$$

for $i \in \Phi_{k}$, and $k \in \mathrm{N}_{\alpha} \cup \alpha$.

Remark 4: Is should be noted that only accessibility is important for defining the concept of unobservability subspaces and the exact values of the transition probabilities do not affect an unobservability subspace. However, the outer MSdetectability of a subspace does depend on the values of the transition probabilities. Moreover, in Section VI outer MSdetectability is shown for systems where uncertainties exist in the transition probabilities.

\section{B. Unobservability Subspace for the MJLSD's}

In this section, the concept of the unobservability subspace that was introduced in the previous subsection for the system $(\mathfrak{A}, \mathfrak{C}, \Pi)$ is generalized to the system $\left(\mathfrak{A}, \mathfrak{A}^{\tau}, \mathfrak{C}, \Pi\right)$. We start 
by defining the notion of conditioned invariant subspaces for a class $\Phi_{\alpha}$ of $\left(\mathfrak{A}, \mathfrak{A}^{\tau}, \mathfrak{C}, \Pi\right)$.

Definition 10: A subspace $\mathcal{W}_{\alpha}^{\tau}$ is said to be conditioned invariant for all modes $i \in \Phi_{\alpha}$ of the system $\left(\mathfrak{A}, \mathfrak{A}^{\tau}, \mathfrak{C}, \Pi\right)$ if $A_{i}\left(\mathcal{W}_{\alpha}^{\tau} \cap \operatorname{Ker} C_{i}\right) \subseteq \mathcal{W}_{\alpha}$ and $A_{i}^{\tau}\left(\mathcal{W}_{\alpha}^{\tau} \cap \operatorname{Ker} C_{i}\right) \subseteq \mathcal{W}_{\alpha}^{\tau}, i \in \Phi_{\alpha}$.

We denote the class of conditioned invariant subspaces of a class $\Phi_{\alpha}$ of $\left(\mathfrak{A}, \mathfrak{A}^{\tau}, \mathfrak{C}, \Pi\right)$ by $\mathfrak{W}_{\{\alpha\}}\left(\mathfrak{A}, \mathfrak{A}^{\tau}, \mathfrak{C}\right)$. It follows that if $\mathcal{W}_{\alpha}^{\tau} \in \mathfrak{W}_{\{\alpha\}}\left(\mathfrak{A}, \mathfrak{A}^{\tau}, \mathfrak{C}\right)$, then there exist maps $D_{i}, D_{i}^{\tau}$ such that $\left(A_{i}+D_{i} C_{i}\right) \mathcal{W}_{\alpha}^{\tau} \subseteq \mathcal{W}_{\alpha}^{\tau}$ and $\left(A_{i}^{\tau}+D_{i}^{\tau} C_{i}\right) \mathcal{W}_{\alpha}^{\tau} \subseteq \mathcal{W}_{\alpha}^{\tau}$, $i \in \Phi_{\alpha}$. Similar to the conditioned invariant subspace for the MJLS's, the family of conditioned invariant subspaces of $\Phi_{\alpha}$ for $\left(\mathfrak{A}, \mathfrak{A}^{\tau}, \mathfrak{C}, \Pi\right)$ which contains a given subspace $\mathcal{L}$ (denoted by $\mathfrak{W}_{\{\alpha\}}\left(\mathfrak{A}, \mathfrak{A}^{\tau}, \mathfrak{C}, \mathcal{L}\right)$ ) is closed under the operation of subspace intersection and is nonempty. The following algorithm can be used for obtaining its smallest element $\mathcal{W}_{\alpha}^{\tau^{*}}$.

Algorithm 5: The subspace $\mathcal{W}_{\alpha}^{\tau^{*}}$ is determined from the last term of the following sequence $\mathcal{W}_{k}=\mathcal{L}+$ $\sum_{i \in \Phi_{\alpha}} A_{i}\left(\mathcal{W}_{k-1} \cap \operatorname{Ker} C_{i}\right)+\sum_{i \in \Phi_{\alpha}} A_{i}^{\tau}\left(\mathcal{W}_{k-1} \cap \operatorname{Ker} C_{i}\right)$ with $\mathcal{W}_{0}=\mathcal{L}$

Definition 11: A subspace $\mathcal{S}_{\alpha}^{\tau}$ is a finite unobservability subspace for all modes $i \in \Phi_{\alpha}$ of $\left(\mathfrak{A}, \mathfrak{A}^{\tau}, \mathfrak{C}, \Pi\right)$ if there exist output maps $D_{i}, D_{i}^{\tau}$ and $H_{i}, i \in \Phi_{\alpha}$ such that $\mathcal{S}_{\alpha}^{\tau}$ is a finite unobservable subspace of class $\Phi_{\alpha}$ of the system $\left(\tilde{\mathfrak{A}}, \tilde{\mathfrak{A}}^{\tau}, \tilde{\mathfrak{C}}, \Pi\right)$, where $\tilde{\mathfrak{A}}=\left\{A_{1}+D_{1} C_{1}, \ldots, A_{N}+D_{N} C_{N}\right\}, \tilde{\mathfrak{A}}^{\tau}=\left\{A_{1}^{\tau}+\right.$ $\left.D_{1}^{\tau} C_{1}, \ldots, A_{N}+D_{N}^{\tau} C_{N}\right\}$, and $\mathfrak{C}=\left\{H_{1} C_{1}, \ldots, H_{N} C_{N}\right\}$.

It follows that $\mathcal{S}_{\alpha}^{\tau}=<<\bigcap_{j \in \Phi_{\alpha}} \operatorname{Ker} H_{j} C_{j} \bigcap \mathcal{K}_{\alpha} \mid A_{i}+$ $D_{i} C_{i}, A_{i}^{\tau}+D_{i}^{\tau} C_{i}>>_{i \in \Phi_{\alpha}}$, where if $\Phi_{\alpha}$ is closed, then $\mathcal{K}_{\alpha}=\mathcal{X}$, and if $\Phi_{\alpha}$ is open, then $\mathcal{K}_{\alpha}=\bigcap_{l \in \mathrm{N}_{\alpha}} \mathcal{S}_{l}^{\tau}$.

For a given subspace $\mathcal{L}$, we denote the class of all unobservability subspaces for class $\Phi_{\alpha}$ of $\left(\mathfrak{A}, \mathfrak{A}^{\tau}, \mathfrak{C}, \Pi\right)$ which contains $\mathcal{L}$ by $\mathfrak{S}_{\alpha}\left(\mathfrak{A}, \mathfrak{A}^{\tau}, \mathfrak{C}, \mathcal{L}\right)$. Following the same procedure as in Section IV-A, it can be shown that $\mathfrak{S}_{\alpha}\left(\mathfrak{A}, \mathfrak{A}^{\tau}, \mathfrak{C}, \mathcal{L}\right)$ is closed under operation of subspace intersection. Moreover, the necessary and sufficient conditions for $\mathfrak{S}_{\alpha}\left(\mathfrak{A}, \mathfrak{A}^{\tau}, \mathfrak{C}, \mathcal{L}\right)$ to be nonempty is that there exists $\mathcal{W}_{\alpha}^{\tau} \in \mathfrak{W}_{\{\alpha\}}\left(\mathfrak{A}, \mathfrak{A}^{\tau}, \mathfrak{C}\right)$ such that $\mathcal{W}_{\alpha}^{\tau} \subseteq \mathcal{K}_{\alpha}$. Similar as in Section IV-A, the following algorithm can be used for determining the infimal element $\mathcal{S}_{\alpha}^{\tau^{*}}=\inf \mathfrak{S}_{\alpha}\left(\mathfrak{A}, \mathfrak{A}^{\tau}, \mathfrak{C}, \mathcal{L}\right)$.

Algorithm 6: Let $\mathcal{W}_{\alpha}^{\tau^{*}}=\inf \mathfrak{W}_{\{\alpha\}}\left(\mathfrak{A}, \mathfrak{A}^{\tau}, \mathfrak{C}, \mathcal{L}\right)$ and conditions for non-emptiness of $\mathfrak{S}_{\alpha}\left(\mathfrak{A}, \mathfrak{A}^{\tau}, \mathfrak{C}, \mathcal{L}\right)$ be satisfied. Define the sequence $\mathcal{Z}^{\mu}$ according to

$$
\begin{aligned}
\mathcal{Z}^{0}=\mathcal{X} ; \quad \mathcal{Z}^{\mu} & =\bigcap_{i \in \Phi_{\alpha}}\left(\mathcal{W}_{\alpha}^{\tau^{*}}+\left(A_{i}^{-1} \mathcal{Z}^{\mu-1} \cap \operatorname{Ker} C_{i}\right)\right) \\
& \bigcap_{i \in \Phi_{\alpha}}\left(\mathcal{W}_{\alpha}^{\tau^{*}}+\left(A_{i}^{\tau^{-1}} \mathcal{Z}^{\mu-1} \cap \operatorname{Ker} C_{i}\right)\right) \bigcap \mathcal{K}_{\alpha}
\end{aligned}
$$

Then $\mathcal{S}_{\alpha}^{\tau^{*}}=\mathcal{Z}^{\mu}$, whenever $\mathcal{Z}^{\mu+1}=\mathcal{Z}^{\mu}$.

Remark 5: It should be emphasized that the notion of u.o.s for the MJSLD's only depends on the matrices $A_{i}, A_{i}^{\tau}$ and $C_{i}$ 's and $i s$ not affected by the values of the delays $\tau_{i}, i \in \Psi$ as well as the values of the transition probabilities.

Finally, similar procedure as in Algorithm 4 can be derived for constructing the unobservability subspaces for an open class $\Phi_{\alpha}$. The details are not included due to space limitations. We are now in the position to formally introduce the FPRG for the Markovian jump systems.

\section{Fundamental Problem in Residual Generation}

In this section, the fundamental problem in residual generation (FPRG) is investigated for the MJLS's and the MJLSD's. This problem was originally considered for linear systems in [2] and was extended to LPV [23], bilinear [24], timedelay [25], [26], and nonlinear [31] systems. The objective in this section is to generalize these results to Markovian jump systems with and without time-delays.

\section{A. FPRG for the MJLS's}

Consider the following Markovian jump linear system

$$
\begin{aligned}
& \dot{x}(t)=A_{\lambda(t)} x(t)+B_{\lambda(t)} u(t)+L_{\lambda(t)}^{1} m_{1}(t)+L_{\lambda(t)}^{2} m_{2}(t) \\
& y(t)=C_{\lambda(t)} x(t), \quad x(0)=x_{0}, \quad \lambda(0)=i_{0}
\end{aligned}
$$

where it is assumed that the matrices $A_{\lambda(t)}$ and $C_{\lambda(t)}$ are the same as in (1) and $u \in \mathcal{U}$ is the input with dimension $m$. The matrices $L_{\lambda(t)}^{1}$ and $L_{\lambda(t)}^{2}$ represent the fault signatures and are monic and $m_{i}(t) \in \mathcal{M}_{i} \subset \mathcal{X}, i=1,2$ denote the fault modes. We denote the matrices $B_{\lambda(t)}, L_{\lambda(t)}^{1}$ and $L_{\lambda(t)}^{2}$ that are associated with $\lambda(t)=i$ by $B_{i}, L_{i}^{1}$ and $L_{i}^{2}$, respectively.

Remark 6: The extension of this problem to multiple (that is more than two) faults is trivial and is not included for notational simplicity. Notwithstanding this an $H_{\infty}$-based FDI strategy for the MJLS's is introduced in Section VI which treats the multiple faults scenario.

The FPRG for each initial operational mode $i_{0}$ of system (8) with possible jumps among classes at time instants $\nu_{k}, k=$ $0, \ldots, \varrho, \nu_{0}=0$ is concerned with the design of a Markovian jump residual generator of the form

$$
\begin{aligned}
\dot{w}_{\bar{\lambda}(t)}(t)= & F_{\lambda(t)} w_{\bar{\lambda}(t)}(t)-E_{\lambda(t)} y(t) \\
& +K_{\lambda(t)} u(t), \quad t \neq \nu_{k}, \lambda(t) \in \Phi_{\bar{\lambda}(t)} \\
w_{\bar{\lambda}\left(\nu_{k}\right)}\left(\nu_{k}\right)= & T_{\bar{\lambda}\left(\nu_{k}\right), \bar{\lambda}\left(\nu_{k}^{-}\right)} w_{\bar{\lambda}\left(\nu_{k}^{-}\right)}\left(\nu_{k}^{-}\right), \quad t=\nu_{k} \\
r_{\bar{\lambda}(t)}(t)= & M_{\lambda(t)} w_{\bar{\lambda}(t)}(t)-H_{\lambda(t)} y(t)
\end{aligned}
$$

where $\lambda(0)=i_{0}, w_{\bar{\lambda}(0)}(0)=0, w_{\bar{\lambda}(t)}(t) \in \mathcal{F}_{\bar{\lambda}(t)} \subset \mathcal{X}$ is the continuous-time state of the above detection filter with non-increasing dimension, and $r_{\bar{\lambda}(t)}(t)$ is the residual signal that satisfies the following properties, namely (a) the response of $r_{\bar{\lambda}(t)}(t)$ is affected by the fault mode $m_{1}(t)$ and is decoupled from $m_{2}(t)$, and (b) if $m_{1}$ is identically zero then $\lim _{t \rightarrow \infty} \mathbb{E} \|\left. r_{\bar{\lambda}(t)}(t)\right|^{2}=0$ for any input signal $u(t)$.

Let us define the extended space $\mathcal{X}_{\bar{\lambda}(t)}^{e}=\mathcal{X} \oplus \mathcal{F}_{\bar{\lambda}(t)}$ and $\mathcal{U}^{e}=\mathcal{U} \oplus \mathcal{M}_{2}$, so that equations (8) and (9) can be re-written as follows

$$
\begin{aligned}
\dot{x}_{\bar{\lambda}(t)}^{e}(t)= & A_{\lambda(t)}^{e} x_{\bar{\lambda}(t)}^{e}(t)+B_{\lambda(t)}^{e} u^{e}(t) \\
& +L_{\lambda(t)}^{e 1} m_{1}(t), \quad t \neq \nu_{k}, \lambda(t) \in \Phi_{\bar{\lambda}(t)} \\
r_{\bar{\lambda}(t)}(t)= & H_{\lambda(t)}^{e} x_{\bar{\lambda}(t)}^{e}(t), \quad \lambda(0)=i_{0}
\end{aligned}
$$

where $x_{\bar{\lambda}\left(\nu_{k}\right)}^{e}\left(\nu_{k}\right)=T_{\bar{\lambda}\left(\nu_{k}\right), \bar{\lambda}\left(\nu_{k}^{-}\right)}^{e} x_{\bar{\lambda}\left(\nu_{k}^{-}\right)}^{e}\left(\nu_{k}^{-}\right)$for $t=\nu_{k}$, $x_{\bar{\lambda}(t)}^{e}(t) \in \mathcal{X}^{e}, u^{e} \in \mathcal{U}^{e}, A_{i}^{e}=\left[\begin{array}{cc}A_{i} & 0 \\ -E_{i} C_{i} & F_{i}\end{array}\right], B_{i}^{e}=\left[\begin{array}{cc}B_{i} & L_{i}^{2} \\ K_{i} & 0\end{array}\right]$, $H_{i}^{e}=\left[\begin{array}{ll}-H_{i} C_{i} M_{i}\end{array}\right], L_{i}^{e 1}=\left[\begin{array}{c}L_{i}^{1} \\ 0\end{array}\right]$, and $T_{\bar{\lambda}\left(\nu_{k}\right), \bar{\lambda}\left(\nu_{k}^{-}\right)}^{e}=$ $\left[\begin{array}{cc}I & 0 \\ 0 & T_{\bar{\lambda}\left(\nu_{k}\right), \bar{\lambda}\left(\nu_{k}^{-}\right)}\end{array}\right]$. In order to investigate the criteria for determining whether a nonzero $m_{1}(t)$ affects the residual signal 
$r_{\bar{\lambda}(t)}(t)$, the notion of an input observability for the MJLS's is first defined and formalized below.

Definition 12: The input signal $m_{1}(t)$ is called input observable for a class $\Phi_{\alpha}$ of system (10) if $L_{1 i}^{e}$ is monic and the image of $L_{1 i}^{e}$ does not intersect with the unobservable subspace $\mathcal{S}_{\alpha}^{e}$ for a class $\Phi_{\alpha}$ of system (10).

Based on the above definition, the FPRG can now be formally stated as the problem of designing the dynamical detection filter (9) so that (a) $r_{\bar{\lambda}(t)}(t)$ is decoupled from $u^{e}(t)$, (b) $m_{1}(t)$ is input observable in (10) for $i_{0}$ and all $j$ such that $i_{0} \rightsquigarrow j$, and (c) $\lim _{t \rightarrow 0} \mathbb{E}\left\{\left\|r_{\bar{\lambda}(t)}(t)\right\|^{2}\right\}=0, \quad$ for $m_{1}(t)=$ $0, \forall x_{0}^{e} \in \mathcal{X}^{e}$

We need to first derive a preliminary result for obtaining the solvability condition for the FPRG. The following embedding map $Q_{\bar{\lambda}(t)}: \mathcal{X} \rightarrow \mathcal{X}_{\bar{\lambda}(t)}^{e}$ is defined according to [2], namely $Q_{\bar{\lambda}(t)} x=\left[\begin{array}{l}x \\ 0\end{array}\right]$ where if $\mathcal{V} \subset \mathcal{X}_{\bar{\lambda}(t)}^{e}$, we have $Q_{\bar{\lambda}(t)}^{-1} \mathcal{V}=\{x \in$ $\left.\mathcal{X} \mid\left[\begin{array}{l}x \\ 0\end{array}\right] \in \mathcal{V}\right\}$. We denote $Q_{\bar{\lambda}(t)}$ and $Q_{\bar{\lambda}(t)}^{-1}$ for $\bar{\lambda}(t)=\alpha$ by $Q_{\alpha}$ and $Q_{\alpha}^{-1}$, respectively. Our first result is the generalization of Proposition 1 that was obtained in [2] to the MJLS's (8) and (10).

Lemma 5.1: Let $\mathcal{S}_{\alpha}^{e}$ be the unobservable subspace for a class $\Phi_{\alpha}$ of system (10) and let $\mathcal{S}_{l}^{e}$ be the unobservable subspace for all modes $l \in \Phi_{\alpha}$. It can be concluded that $\mathcal{S}_{\alpha}=Q_{\alpha}^{-1} \mathcal{S}_{\alpha}^{e}$ and $\mathcal{S}_{l}=Q_{l}^{-1} \mathcal{S}_{l}^{e}, l \in \Phi_{\alpha}$ are the unobservability subspaces for $(\mathfrak{A}, \mathfrak{C}, \Pi)$.

Proof: Consider the unobservable subspace of system (10) with $i_{0} \in \Phi_{\alpha}$ (denoted as $\mathcal{S}_{\alpha}^{e}$ ). Due to the fact that the initial condition of the detection filter in (9) is known, we can write $\mathcal{S}_{\alpha}^{e}=\left[\begin{array}{c}\mathcal{S}_{\alpha} \\ 0\end{array}\right]$. Following the results in Section III-A, it is known that for all $i$ in $\Phi_{\alpha}, \mathcal{S}_{\alpha}^{e}$ is $A_{i}^{e}$-invariant. This is due to the fact that while system (10) remains in class $\Phi_{\alpha}$, the dimension of the system is fixed and the results of Section III-A can be used. Moreover, when $\bar{\lambda}(t)$ leaves the class $\Phi_{\alpha}$ and enters another class $\Phi_{l}$ then $T_{l, \alpha}^{e} \mathcal{S}_{\alpha}^{e} \subseteq \mathcal{S}_{l}^{e}$, where $\mathcal{S}_{l}^{e}$ is an unobservable subspace for class $\Phi_{l}$ which has a similar structure as that of $\mathcal{S}_{\alpha}^{e}$, i.e. $\mathcal{S}_{l}^{e}=\left[\begin{array}{c}\mathcal{S}_{l} \\ 0\end{array}\right]$. However, we have $T_{l, \alpha}^{e} \mathcal{S}_{\alpha}^{e}=\left[\begin{array}{c}\mathcal{S}_{\alpha} \\ 0\end{array}\right]$, and consequently, $\mathcal{S}_{\alpha} \subseteq \bigcap_{i \in \mathrm{N}_{\alpha}} \mathcal{S}_{l}$.

Next, we show that for each class $\Phi_{\alpha}$ the subspace $\mathcal{S}_{\alpha}=$ $Q_{\alpha}^{-1} \mathcal{S}_{\alpha}^{e}$ is conditioned invariant for the class $\Phi_{\alpha}$. Let $x \in$ $\mathcal{S}_{\alpha} \cap \operatorname{Ker} C_{i}$, therefore one needs to show that $A_{i} x \in \mathcal{S}_{\alpha}, i \in$ $\Phi_{\alpha}$. This follows by noting that $\left[\begin{array}{c}A_{i} x \\ 0\end{array}\right]=\left[\begin{array}{cc}A_{i} & 0 \\ -E_{i} C_{i} & F_{i}\end{array}\right]\left[\begin{array}{l}x \\ 0\end{array}\right] \in$ $\mathcal{S}_{\alpha}^{e}$, since $\mathcal{S}_{\alpha}^{e}$ is $A_{i}^{e}$-invariant with $i \in \Phi_{\alpha}$. Therefore, $A_{i} x \in$ $\mathcal{S}_{\alpha}$ and $\mathcal{S}_{\alpha}$ is conditioned invariant. Now if $x \in \mathcal{S}_{\alpha}$, then $Q_{\alpha} x \in \mathcal{S}_{\alpha}^{e}$, and therefore $\left[\begin{array}{c}x \\ 0\end{array}\right] \in \bigcap_{i \in \Phi_{\alpha}} \operatorname{Ker} H_{i}^{e}$. This shows that $H_{i} C_{i} x=0$; and hence $x \in \bigcap_{i \in \Phi_{\alpha}} \operatorname{Ker} H_{i} C_{i}$. It follows that for the closed class $\Phi_{\alpha}$, we have $\mathcal{S}_{\alpha} \subseteq \bigcap_{j \in \Phi_{\alpha}} \operatorname{Ker} H_{j} C_{j}$. Hence, according to the definition of the unobservable subspace $\mathcal{S}_{\alpha}^{e}$ (the largest $A_{i}^{e}$-invariant subspace in $\bigcap_{j \in \Phi_{\alpha}} \operatorname{Ker} H_{i}^{e}$ ), $\mathcal{S}_{\alpha}$ is the largest conditioned invariant subspace contained in $\bigcap_{i \in \Phi_{\alpha}} \operatorname{Ker} H_{i} C_{i}$, and therefore $\mathcal{S}_{\alpha} \in \mathfrak{S}_{\alpha}(\mathfrak{A}, \mathfrak{C})$.

For an open class $\Phi_{\alpha}$, note that for all the closed class $\Phi_{j}, j \in \mathbf{N}_{\alpha}^{c}$, it is shown above that $\mathcal{S}_{j}=Q_{j}^{-1} \mathcal{S}_{j}^{e}$ is an u.o.s. for the class $\Phi_{j}$. Therefore, let us start with $\Phi_{\alpha_{1}^{o}}$ (the first open class in $\mathrm{N}_{\alpha}^{o}$ ). It follows that all the classes in $\mathrm{N}_{\alpha_{1}^{o}}$ are closed. Moreover, one has $\mathcal{S}_{\alpha_{1}^{o}} \subseteq \bigcap_{j \in \mathrm{N}_{\alpha_{1}^{o}}} \mathcal{S}_{j}$. Therefore, $\mathcal{S}_{\alpha_{1}^{o}} \subseteq \bigcap_{j \in \Phi_{\alpha_{1}^{o}}} \operatorname{Ker} H_{j} C_{j} \bigcap_{j \in \mathrm{N}_{\alpha_{1}^{o}}} \mathcal{S}_{j}$. Similarly, one can show that $\mathcal{S}_{\alpha_{1}^{o}}$ is the largest conditioned invariant subspace contained in $\bigcap_{j \in \Phi_{\alpha_{1}^{o}}} \operatorname{Ker} H_{j} C_{j} \bigcap_{j \in \mathrm{N}_{\alpha_{1}^{o}}} \mathcal{S}_{j}$. Following along similar lines one can show that for all open classes $\Phi_{\alpha_{j}^{o}}$ as well as $\Phi_{\alpha}$, one gets $\mathcal{S}_{\alpha}=Q_{\alpha}^{-1} \mathcal{S}_{\alpha}^{e}$ as an u.o.s.

We are now in the position to derive the solvability conditions for the FPRG corresponding to the MJLS (10).

Theorem 5.2: The FPRG has a solution for the augmented MJLS (10) and initial mode $i_{0} \in \Phi_{\alpha}$ only if $\mathcal{S}_{\alpha}^{*} \cap \mathcal{L}_{j}^{1}=0$, $j \in \Phi_{\alpha}$, where $\mathcal{S}_{\alpha}^{*}=\inf \mathfrak{S}_{\alpha}\left(\mathfrak{A}, \mathfrak{C}, \sum_{i \in \Phi_{\alpha}} \mathcal{L}_{i}^{2}\right)$ and for an open class $\Phi_{\alpha}, \mathcal{S}_{l}^{*} \cap \mathcal{L}_{j}^{1}=0, j \in \Phi_{l}, l \in \mathbf{N}_{\alpha}$, where $\mathcal{S}_{l}^{*}=\inf \mathfrak{S}_{l}\left(\mathfrak{A}, \mathfrak{C}, \mathcal{L}_{l}\right)$ and $\mathcal{L}_{l}=\sum_{i \in \Phi_{l} \cup \Phi_{\alpha}} \mathcal{L}_{i}^{2}+$ $\sum_{j \in \Phi_{k}, k \in \mathrm{N}_{\alpha}, l \in \mathrm{N}_{k}} \mathcal{L}_{j}^{2}$. On the other hand, if $\mathcal{S}_{\alpha}^{*}$ and $\mathcal{S}_{l}^{*}, l \in \mathrm{N}_{\alpha}$ (if $\Phi_{\alpha}$ is open) exist such that they are also outer MSdetectable, then the FPRG has a solution for the initial mode $i_{0} \in \Phi_{\alpha}$ of the MJLS (10).

Proof: (Only if part) Let $\mathcal{S}_{\alpha}^{e}$ be an unobservable subspace of system (10). To satisfy the condition (a) of the FPRG, we should have $\mathcal{B}_{i}^{e} \subset \mathcal{S}_{\alpha}^{e}, i \in \Phi_{\alpha}$ and $\mathcal{B}_{j}^{e} \subset \mathcal{S}_{l}^{e}, j \in$ $\Phi_{l}, l \in \mathrm{N}_{\alpha}$. Hence, $\mathcal{L}_{i}^{2} \subset Q_{\alpha}^{-1} \mathcal{B}_{i}^{e} \subset Q_{\alpha}^{-1} \mathcal{S}_{\alpha}^{e}=\mathcal{S}_{\alpha}$ and $\mathcal{L}_{j}^{2} \subset Q_{l}^{-1} \mathcal{B}_{j}^{e} \subset Q_{l}^{-1} \mathcal{S}_{l}^{e}=\mathcal{S}_{l}$. By invoking Lemma 5.1, we obtain $\mathcal{S}_{\alpha} \in \mathfrak{S}_{\alpha}\left(\mathfrak{A}, \mathfrak{C}, \sum_{i \in \Phi_{\alpha}} \mathcal{L}_{i}^{2}\right)$. Moreover, for any class $\Phi_{l}, l \in \mathrm{N}_{\alpha} \cup \alpha$, it is shown in Lemma 5.1 that $\mathcal{S}_{j} \subseteq \mathcal{S}_{l}$, $j \in \mathrm{N}_{\alpha}, l \in \mathrm{N}_{j}$, and hence $\sum_{j \in \Phi_{k}, k \in \mathrm{N}_{\alpha}, l \in \mathrm{N}_{k}} \mathcal{L}_{j}^{2} \subset \mathcal{S}_{l}$. Therefore, $\mathcal{S}_{l} \in \mathfrak{S}_{l}\left(\mathfrak{A}, \mathfrak{C}, \mathcal{L}_{l}\right)$. For condition (b) of the FPRG to hold, according to the Definition $12, L_{i}^{e 1}$ should be monic for all modes accessible from $\alpha$ (which is already assumed to hold) and $\mathcal{L}_{i}^{e 1} \cap \mathcal{S}_{\alpha}^{e}=0, i \in \Phi_{\alpha}$ and $\mathcal{L}_{j}^{e 1} \cap \mathcal{S}_{l}^{e}=0, j \in \Phi_{l}$ and $l \in \mathrm{N}_{\alpha}$. Therefore, $Q_{\alpha}^{-1}\left(\mathcal{L}_{i}^{e 1} \cap \mathcal{S}_{\alpha}^{e}\right)=\mathcal{L}_{i}^{1} \cap \mathcal{S}_{\alpha}=0$, $i \in \Phi_{\alpha}$ and $Q_{l}^{-1}\left(\mathcal{L}_{j}^{e 1} \cap \mathcal{S}_{l}^{e}\right)=\mathcal{L}_{j}^{1} \bigcap \mathcal{S}_{l}=0, j \in \Phi_{l}, l \in \mathrm{N}_{\alpha}$. Consequently, the above conditions hold only if $\mathcal{S}_{\alpha}^{*} \cap \mathcal{L}_{j}^{1}=0$, $j \in \Phi_{\alpha}$, where $\mathcal{S}_{\alpha}^{*}=\inf \mathfrak{S}_{\alpha}\left(\mathfrak{A}, \mathfrak{C}, \sum_{i \in \Phi_{\alpha}} \mathcal{L}_{i}^{2}\right)$ and for an open class $\Phi_{\alpha}, \mathcal{S}_{l}^{*} \cap \mathcal{L}_{j}^{1}=0, j \in \Phi_{l}, l \in \mathbf{N}_{\alpha}$, where $\mathcal{S}_{l}^{*}=\inf \mathfrak{S}_{l}\left(\mathfrak{A}, \mathfrak{C}, \mathcal{L}_{l}\right)$.

(if part): First, we consider the case where $\Phi_{\alpha}$ is a closed class. Given the unobservability subspace $\mathcal{S}_{\alpha}^{*}$ which is outer MS-detectable, there exist $D_{i}$ and $H_{i}, i \in \Phi_{\alpha}$ such that $\mathcal{S}_{\alpha}^{*}=<<\bigcap_{j \in \Phi_{\alpha}} \operatorname{Ker} H_{j} C_{j} \mid A_{i}+D_{i} C_{i}>>_{i \in \Phi_{\alpha}}$. Let $P_{\alpha}$ be the canonical projection of $\mathcal{X}$ on $\mathcal{X} / \mathcal{S}_{\alpha}^{*}$ and $M_{i}, i \in \Phi_{\alpha}$ be a unique solution to $M_{i} P_{\alpha}=H_{i} C_{i}$ and $\bar{A}_{i}=\left(A_{i}+D_{i} C_{i}\right.$ : $\left.\mathcal{X} / \mathcal{S}_{\alpha}^{*}\right)$, where $P_{\alpha}\left(A_{i}+D_{i} C_{i}\right)=\bar{A}_{i} P_{\alpha}, i \in \Phi_{\alpha}$. Due to the fact that $\mathcal{S}_{\alpha}^{*}$ is assumed to be outer MS-detectable, there exist $G_{i}, i \in \Phi_{\alpha}$ such that $\left(\overline{\mathfrak{A}}_{G}, \Pi\right)$ is MS-stable, where $\overline{\mathfrak{A}}_{G}=\left\{\bar{A}_{j}+G_{j} M_{j}, j \in \Phi_{\alpha}\right\}$.

Let us define $F_{i}=\bar{A}_{i}+G_{i} M_{i}, E_{i}=P_{\alpha}\left(D_{i}+P_{\alpha}^{-r} G_{i} H_{i}\right)$, $K_{i}=P_{\alpha} B_{i}, \quad i \in \Phi_{\alpha}$, and $e_{\alpha}(t)=w_{\alpha}(t)-P_{\alpha} x(t)$. By invoking equation (9) for $\bar{\lambda}(t)=\alpha$ and $\varrho=0$ (no jumps among classes since $\Phi_{\alpha}$ is closed) we obtain

$$
\begin{aligned}
\dot{e}_{\alpha}(t)= & F_{i} w_{\alpha}(t)-E_{i} y(t)+K_{i} u(t) \\
& \left.-P_{\alpha}\left(A_{i} x(t)+B_{i} u(t)+L_{i}^{1} m_{1}(t)\right)+L_{i}^{2} m_{2}(t)\right) \\
= & F_{i} w_{\alpha}(t)-P_{\alpha} L_{i}^{1} m_{1}(t) \\
& -P_{\alpha}\left(A_{i}+D_{i} C_{i}\right) x(t)-G_{i} H_{i} C_{i} x(t) \\
= & F_{i} w_{\alpha}(t)-P_{\alpha} L_{i}^{1} m_{1}(t)-\bar{A}_{i} P_{\alpha} x(t)-G_{i} M_{i} P_{\alpha} x(t) \\
= & F_{i} e_{\alpha}(t)-P L_{i}^{1} m_{1}(t)
\end{aligned}
$$

Note that $P_{\alpha} L_{i}^{2}=0, i \in \Phi_{\alpha}$, since $\mathcal{L}_{i}^{2} \subset \mathcal{S}_{\alpha}^{*}, i \in \Phi_{\alpha}$. Also, $r_{\alpha}(t)=M_{i} w_{\alpha}(t)-H_{i} y(t)=M_{i} w_{\alpha}(t)-H_{i} C_{i} x(t)=$ $M_{i} e_{\alpha}(t)$. Consequently, the error dynamics can be written 
according to

$$
\begin{aligned}
& \dot{e}_{\alpha}(t)=F_{\lambda(t)} e_{\alpha}(t)-P_{\alpha} L_{\lambda(t)}^{1} m_{1}(t) \\
& r_{\alpha}(t)=M_{\lambda(t)} e_{\alpha}(t), \lambda(t) \in \Phi_{\alpha}
\end{aligned}
$$

It follows that the fault mode $m_{2}(t)$ does not affect the residual signal $r_{\alpha}(t)$ and since the dynamics (11) is observable, condition (b) of the FPRG also holds. Moreover, for $m_{1}(t)=0$ the system is MS-stable and $\lim _{t \rightarrow 0} \mathbb{E}\left\{\left\|r_{\alpha}(t)\right\|^{2}\right\}=0$.

Next, we consider the case where $\Phi_{\alpha}$ is an open class. Given the unobservability subspaces $\mathcal{S}_{\alpha}^{*}$ and $\mathcal{S}_{l}^{*}, l \in \Phi_{\alpha}$ which are outer MS-detectable, there exist $D_{i}$ and $H_{i}, i \in \Phi_{\alpha}$ and $D_{j}$ and $H_{j}, j \in \Phi_{l}, l \in \mathrm{N}_{\alpha}$ such that $\mathcal{S}_{\alpha}^{*}=<<$ $\bigcap_{j \in \Phi_{\alpha}} \operatorname{Ker} H_{j} C_{j} \bigcap_{l \in \mathrm{N}_{\alpha}} \mathcal{S}_{l} \mid A_{i}+D_{i} C_{i}>>_{i \in \Phi_{\alpha}}$ and $\mathcal{S}_{l}^{*}=<<$ $\bigcap_{j \in \Phi_{l}} \operatorname{Ker} H_{j} C_{j} \bigcap_{k \in \mathrm{N}_{l}} \mathcal{S}_{k} \mid A_{i}+D_{i} C_{i}>>_{i \in \Phi_{l}}$ if $\Phi_{l}$ is open and $\mathcal{S}_{l}=<<\bigcap_{j \in \Phi_{l}} \operatorname{Ker} H_{j} C_{j} \mid A_{i}+D_{i} C_{i}>_{i \in \Phi_{l}}$ if $\Phi_{l}$ is closed. Let $P_{\alpha}$ and $P_{l}$ be the canonical projections of $\mathcal{X}$ on $\mathcal{X} / \mathcal{S}_{\alpha}^{*}$ and $\mathcal{X} / \mathcal{S}_{l}^{*}$, respectively and $T_{l, \alpha}$ be the canonical projection of $\mathcal{X} / \mathcal{S}_{\alpha}^{*}$ on $\mathcal{X} / \mathcal{S}_{l}^{*}$.

Let $M_{i}, i \in \Phi_{\alpha}$ be a unique solution to $M_{i} P_{\alpha}=H_{i} C_{i}$ and $\bar{A}_{i}=\left(A_{i}+D_{i} C_{i}: \mathcal{X} / \mathcal{S}_{\alpha}^{*}\right)$, where $P_{\alpha}\left(A_{i}+D_{i} C_{i}\right)=$ $\bar{A}_{i} P_{\alpha}, i \in \Phi_{\alpha}$. Moreover, the maps $M_{j}$ and $\bar{A}_{j}=\left(A_{j}+\right.$ $\left.D_{j} C_{j}: \mathcal{X} / \mathcal{S}_{l}\right), j \in \Phi_{l}, l \in \mathrm{N}_{\alpha}$ can be found from expressions $M_{j} P_{l}=H_{j} C_{j}$ and $P_{l}\left(A_{j}+D_{j} C_{j}\right)=\bar{A}_{j} P_{l}$, respectively. Due to the fact that $\mathcal{S}_{\alpha}^{*}$ and $\mathcal{S}_{l}^{*}$ are assumed to be outer MSdetectable, there exist $G_{i}, i \in \Phi_{\alpha}$ and $G_{j}, j \in \Phi_{l}, l \in \mathrm{N}_{\alpha}$ such that system $\left(\overline{\mathfrak{A}}_{G}, \Pi\right)$ is MS-stable where $\overline{\mathfrak{A}}_{G}=\left\{A_{i}+\right.$ $\left.G_{i} M_{i}, A_{j}+G_{j} M_{j}, i \in \Phi_{\alpha}, j \in \Phi_{l}, l \in \mathrm{N}_{\alpha}\right\}$. Let us define $F_{i}=\bar{A}_{i}+G_{i} M_{i}, E_{i}=P_{\alpha}\left(D_{i}+P_{\alpha}^{-r} G_{i} H_{i}\right), K_{i}=P_{\alpha} B_{i}, i \in$ $\Phi_{\alpha}, F_{j}=\bar{A}_{j}+G_{j} M_{j}, E_{j}=P_{\alpha}\left(D_{j}+P_{\alpha}^{-r} G_{j} H_{j}\right), K_{j}=$ $P_{l} B_{j}, j \in \Phi_{l}, e_{\alpha}(t)=w_{\alpha}(t)-P_{\alpha} x(t)$ and $e_{l}(t)=w_{l}(t)-$ $P_{l} x(t)$. Following along the similar lines as in the case for the closed class $\Phi_{\alpha}$, the error dynamics for $e_{\alpha}(t)$ can be written as

$$
\begin{aligned}
& \dot{e}_{\alpha}(t)=F_{\lambda(t)} e_{\alpha}(t)-P_{\alpha} L_{\lambda(t)}^{1} m_{1}(t) \\
& r_{\alpha}(t)=M_{\lambda(t)} e_{\alpha}(t)
\end{aligned}
$$

as long as $\lambda(t) \in \Phi_{\alpha}$. When $\lambda(t)$ leaves the class $\Phi_{\alpha}$ at $t=\nu_{1}$ and enters another class $\Phi_{l}$, the error dynamics for $e_{l}(t)$ can be expressed as follows

$$
\begin{aligned}
& \dot{e}_{l}(t)=F_{\lambda(t)} e_{l}(t)-P_{l} L_{\lambda(t)}^{1} m_{1}(t) \\
& r_{l}(t)=M_{\lambda(t)} e_{l}(t)
\end{aligned}
$$

where we have $e_{l}\left(\nu_{1}\right)=T_{l, \alpha} e_{\alpha}\left(\nu_{1}\right)$. Similarly, if $\Phi_{l}$ is an open class, once $\lambda(t)$ leaves $\Phi_{l}$ and enters $\Phi_{k}$, the error dynamics for $e_{k}(t)=w_{k}(t)-P_{k} x(t)$ can be found similar to (13). Consequently, the fault mode $m_{2}(t)$ does not affect the residual signal $r_{\bar{\lambda}(t)}(t)$ and the terms $P_{\alpha} L_{\lambda(t)}^{1}$ and $P_{l} L_{\lambda(t)}^{1}$ are nonzero. Moreover, for $m_{1}(t)=0$ the error dynamics are MS-stable and $\lim _{t \rightarrow 0} \mathbb{E}\left\{\left\|r_{\bar{\lambda}(t)}(t)\right\|^{2}\right\}=0$. This completes the proof of the theorem.

Based on the results in Theorem 5.2, for the case when $\Phi_{\alpha}$ is an open class, the detection filer in (9) has different dimensions as specified by $\mathcal{S}_{\alpha}^{*}$ when $\lambda(t) \in \Phi_{\alpha}$, and as specified by $\mathcal{S}_{l}^{*}$ when $\lambda(t) \in \Phi_{l}$. Moreover, when the filter is initiated from mode $i_{0} \in \Phi_{\alpha}$, the dimensions of the filters are non-increasing due to the fact that $\mathcal{S}_{\alpha}^{*} \subseteq \mathcal{S}_{l}^{*}$. Whenever $\lambda(t)$ leaves a class $\Phi_{l}$ and enters $\Phi_{j}$ at time $t=\nu_{k}$, the initial conditions of the filters $w_{j}\left(\nu_{k}\right)$ can be found from the elements of $w_{l}\left(\nu_{k}^{-}\right)$as $w_{j}\left(\nu_{k}\right)=T_{j, l} w_{l}\left(\nu_{k}^{-}\right)$. However, for the closed class $\Phi_{\alpha}$, the dimension of the filer is fixed and is specified by $\mathcal{S}_{\alpha}^{*}$.

Example 2: Consider the MJLS (8) with $N=4$ and matrices $A_{1}=\left[\begin{array}{cccc}-3 & 2 & 0 & 1 \\ -1 & 0 & 2 & 0 \\ -3 & 3 & 1 & -1\end{array}\right], A_{2}=\left[\begin{array}{cccc}1 & 0 & -1 & 1 \\ 0 & 3 & -7 & 2 \\ 0 & 0 & -2 & 0 \\ 1 & 2 & 0 & 3\end{array}\right], A_{3}=\left[\begin{array}{cccc}-4 & 1 & 1 & -1 \\ -1 & 2 & 2 & 1 \\ -3 & -2 & 2 & -3 \\ 2 & 1 & 3 & 6\end{array}\right]$, $A_{4}=\left[\begin{array}{cccc}2 & 1 & -5 & 3 \\ -6 & 3 & -8 & 2 \\ 1 & 4 & -1 & 0 \\ 2 & 1 & 0 & 1\end{array}\right], C_{1}=C_{2}=\left[\begin{array}{cccc}1 & -1 & 0 & 0 \\ 0 & 1 & 1 & 0 \\ 1 & 2 & 0 & 1\end{array}\right], C_{3}=C_{4}=$ $\left[\begin{array}{cccc}2 & -2 & 1 & 1 \\ 1 & 2 & 4 & 2 \\ 3 & 1 & 1 & 1\end{array}\right], L_{i}^{1}=\left[\begin{array}{c}3 \\ 4 \\ -2 \\ 1\end{array}\right], i \in\{1, \ldots, 4\}, L_{1}^{2}=L_{2}^{2}=\left[\begin{array}{c}1 \\ 0 \\ 2 \\ 0\end{array}\right]$, $L_{3}^{2}=L_{4}^{2}=\left[\begin{array}{l}0 \\ 1 \\ 2 \\ 1\end{array}\right], B_{i}=0$, and $\Pi=\left[\begin{array}{cccc}-1 & 1 & 0 & 0 \\ 2 & -2 & 0 & 0 \\ 1 & 0 & -3 & 2 \\ 0 & 2 & 2 & -4\end{array}\right]$. It follows that there exist two classes $\Phi_{1}=\{1,2\}$ and $\Phi_{2}=\{3,4\}$ which are closed and open, respectively. It is assumed that the initial mode is 4. According to Theorem 5.2, one first needs to find $\mathcal{S}_{2}^{*}=\inf \mathfrak{S}_{2}\left(\mathfrak{A}, \mathfrak{C}, L_{3}^{2}+L_{4}^{2}\right)$. Since $\Phi_{2}$ is an open class, one is also required to find $\mathcal{S}_{1}^{*}$ such that $\sum_{i=1}^{4} L_{i}^{2} \subset \mathcal{S}_{1}^{*}$. According to Algorithm $4, \mathcal{S}_{1}$ can be found be setting $\mathcal{Z}_{0}=\sum_{i=1}^{4} \mathcal{L}_{i}^{2}$ which will result in $\mathcal{S}_{1}^{*}=\mathcal{L}_{1}^{2}+\mathcal{L}_{3}^{2}$. Consequently, $\mathcal{S}_{2}^{*}$ can be found by invoking Algorithm 3 by setting $\mathcal{K}_{2}=\mathcal{S}_{1}^{*}$, which yields $\mathcal{S}_{2}^{*}=L_{3}^{2}$. Therefore, $\mathcal{S}_{1}^{*} \bigcap \mathcal{L}_{i}^{1}=0, i=1,2$ and $\mathcal{S}_{2}^{*} \bigcap \mathcal{L}_{i}^{1}=0, i=3,4$, and consequently the FPRG has a solution for the above system provided that $\mathcal{S}_{1}^{*}$ and $\mathcal{S}_{2}^{*}$ are also outer MS-detectable. The detection filters can be designed by following the steps that are provided in the proof of Theorem 5.2. It should be noted that according to $\mathcal{S}_{1}^{*}$ and $\mathcal{S}_{2}^{*}$, the dimension of the filters for $\lambda(t) \in \Phi_{2}$ and $\lambda(t) \in \Phi_{1}$ is 3 and 2, respectively. The map $T_{1,2}$ can also be easily found from the canonical maps $P_{1}$ and $P_{2}$ as $T_{1,2}=P_{1} P_{2}^{-r}$. The details are omitted due to space limitations.

\section{B. FPRG for the MJLSD}

Consider the following MJLSD

$$
\begin{aligned}
\dot{x}(t)= & A_{\lambda(t)} x(t)+A_{\lambda(t)}^{\tau} x\left(t-\tau_{\lambda(t)}(t)\right) \\
& +B_{\lambda(t)} u(t)+L_{\lambda(t)}^{1} m_{1}(t)+L_{\lambda(t)}^{2} m_{2}(t) \\
y(t)= & C_{\lambda(t)} x(t) \quad x(\theta)=\phi(\theta), \theta \in[-\mu, 0], \quad i_{0} \in \Psi
\end{aligned}
$$

where all the matrices are the same as in the dynamical model (2) and $u \in \mathcal{U}$ is the input with dimension $m$. The matrices $L_{\lambda(t)}^{1}$ and $L_{\lambda(t)}^{2}$ represent the fault signatures and are monic and $m_{i}(t) \in \mathcal{M}_{i} \subset \mathcal{X}, i=1,2$ denote the fault modes.

In system (14), $\tau_{\lambda(t)}(t)$ denotes a time-varying delay when the mode is in $\lambda(t)$ and satisfies $0<\tau_{i}(t) \leq \mu_{i}<\infty, \dot{\tau}_{i}(t) \leq$ $h_{i}<1, \forall i \in \Psi$, where $\mu_{i}$ and $h_{i}$ are real constant scalars for any $i \in \Psi$ and $\mu:=\max \left\{\mu_{i}, i \in \Psi\right\}$. In the following, it is assumed that $i_{0}$ belongs to a closed class $\Phi_{\alpha}$. Similar results can be derived for an open class following along the same steps as in Section V-A. These results are omitted due to space limitations.

The FPRG for the MJLSD (14) is concerned with design of a residual generator that is governed by the detection filter dynamics of the form

$$
\begin{aligned}
\dot{w}(t)= & F_{\lambda(t)} w(t)+F_{\lambda(t)}^{\tau} w\left(t-\tau_{\lambda(t)}(t)\right) \\
& -E_{\lambda(t)} y(t)-E_{\lambda(t)}^{\tau} y\left(t-\tau_{\lambda(t)}(t)\right)+K_{\lambda(t)} u(t) \\
r(t)= & M_{\lambda(t)} w(t)-H_{\lambda(t)} y(t)
\end{aligned}
$$


such that (a) the response of $r(t)$ is affected by the fault mode $m_{1}(t)$ and is decoupled from $m_{2}(t)$, and (b) if $m_{1}$ is identically zero then $\lim _{t \rightarrow \infty} \mathbb{E}\|r(t)\|^{2}=0$ for any input signal $u(t)$. The MS-detectability for the MJLSD's is defined next.

Definition 13: We say that the system $\left(\mathfrak{A}, \mathfrak{A}^{\tau}, \mathfrak{C}, \Pi\right)$ is MS-detectable when there exist $\mathfrak{G}=\left(G_{1}, \ldots, G_{N}\right)$ and $\mathfrak{G}^{\tau}=\left(G_{1}^{\tau}, \ldots, G_{N}^{\tau}\right)$ of appropriate dimensions for which system $\left(\mathfrak{A}_{\mathfrak{G}}, \mathfrak{A}_{\mathfrak{G}}^{\tau}, \Pi\right)$ is MS-stable, where $\mathfrak{A}_{\mathfrak{G}}=\left\{A_{1}+\right.$ $\left.G_{1} C_{1}, \ldots, A_{N}+G_{N} C_{N}\right\}$ and $\mathfrak{A}_{\mathfrak{G}}^{\tau}=\left\{A_{1}^{\tau}+G_{1}^{\tau} C_{1}^{\tau}, \ldots, A_{N}^{\tau}+\right.$ $\left.G_{N}^{\tau} C_{N}^{\tau}\right\}$.

The following LMIs can be used for testing the MSdetectability of a MJLSD [13], namely

$$
\left[\begin{array}{cc}
J_{i}+\sum_{j=1}^{N} \pi_{i j} X_{j}+(1+\eta \mu) Q & X_{i} A_{i}^{\tau}+L_{i}^{\tau} C_{i} \\
* & -\left(1-h_{i}\right) Q
\end{array}\right]<0
$$

where $J_{i}=A_{i}^{\prime} X_{i}+X_{i} A_{i}+L_{i} C_{i}+C_{i}^{\prime} L_{i}, \eta=\max \left\{\left|\pi_{i i}\right|, i \in\right.$ $\Psi\}$ and the unknowns $X_{i}, Q>0, L_{i}$, and $L_{i}^{\tau}$ having appropriate dimensions.

The next theorem provides sufficient conditions for solvability of the FPRG for system (14).

Theorem 5.3: The FPRG has a solution to the Markovian jump system (14) with mode-dependent time-delays and initial mode $i_{0} \in \Phi_{\alpha}$ ( $\Phi_{\alpha}$ is closed) if there exists outer MS-detectable unobservability subspace $\mathcal{S}_{\alpha}^{*}=$ $\inf \mathfrak{S}_{\alpha}\left(\mathfrak{A}, \mathfrak{A}^{\tau}, \mathfrak{C}, \sum_{i \in \Phi_{\alpha}} \mathcal{L}_{i}^{2}\right)$ such that $\mathcal{S}_{\alpha}^{*} \cap \mathcal{L}_{i}^{1}=0, i \in \Phi_{\alpha}$.

Proof: The proof is immediate by following along the same lines as that we have already developed for the proof of the sufficient condition in Theorem 5.2, and is therefore omitted due to space limitations.

Remark 7: It should be noted that Theorem 5.3 provides the sufficient conditions for solvability of the FPRG for the MJLSD's. The advantage of this result is that it only uses finite dimensional tools and avoids the complexity of dealing with infinite dimensional systems.

\section{VI. $H_{\infty}$-BASEd Fault Detection AND Isolation DESIGN FOR THE MJLS'S}

In this section, we consider Markovian jump systems that are subjected to both external input and output disturbances and that are governed by

$$
\begin{aligned}
& \dot{x}(t)=A_{\lambda(t)} x(t)+B_{\lambda(t)} u(t)+\sum_{j=1}^{k} L_{\lambda(t)}^{j} m_{j}(t)+B_{\lambda(t)}^{d} d(t) \\
& y(t)=C_{\lambda(t)} x(t)+D_{\lambda(t)}^{d} d(t)
\end{aligned}
$$

where all the matrices are defined as in (8), $L_{\lambda(k)}^{j}, j \in \mathbf{k}$ are the fault signatures, and $m_{j}(t) \in \mathcal{M}_{j}, j \in \mathbf{k}$ are the fault modes. The signal $d(t) \in \mathbb{R}^{p}$ represents an unknown additive disturbance at the input and output channels. We denote the disturbance matrices $B_{\lambda(t)}^{d}$ and $D_{\lambda(t)}^{d}$ and the fault signatures $L_{\lambda(t)}^{j}$ associated with $\lambda(t)=i$ by $B_{d i}, D_{d i}, i \in \mathbf{N}$ and $L_{i}^{j}, i \in \mathbf{N}, j \in \mathbf{k}$, respectively. It is further assumed that the disturbance input $d(t)$ belongs to $\mathscr{L}_{2}[0, \infty]$. It should be noted that similar results can be derived for the MJLSD. However, due to space limitations, only the results for the MJLS's without time-delays is considered in this section. Moreover, for the same reason it is assumed that $\lambda(t)$ is irreducible. Similar results can be derived for a general Markov process $\lambda(t)$ by partitioning it into closed and open classes as in Section V-A. Based on the above assumption, there exists only one closed class $\Phi_{1}=\Psi$.

It is assumed that the mode transition matrix $\Pi$ is not known precisely. In other words, it belongs to the following admissible uncertainty domain [33], namely

$$
\mathscr{D}_{\Pi}=\left\{\bar{\Pi}+\Delta \Pi:\left|\Delta \pi_{i j}\right| \leq \epsilon_{i j}, \epsilon_{i j} \geq 0, \quad \forall i, j \in \mathbf{N}, i \neq j\right\}
$$

where $\bar{\Pi}=\left[\bar{\pi}_{i j}\right]$ is a known constant matrix and denotes the estimated value of $\Pi$, and $\Delta \Pi=\left[\Delta \pi_{i j}\right]$ denotes the uncertainty in the mode transition rate matrix. Based on the above formulation of the MJLS (17), an $H_{\infty}$-based Extended Fundamental Problem in Residual Generation (HEFPRG) is introduced now which is concerned with the design of a set of detection filters

$$
\begin{aligned}
\dot{w}_{j}(t) & =F_{\lambda(t)}^{j} w_{j}(t)-E_{\lambda(t)}^{j} y(t)+K_{\lambda(t)}^{j} u(t) \\
r_{j}(t) & =M_{\lambda(t)}^{j} w_{j}(t)-H_{\lambda(t)}^{j} y(t), \quad j \in \mathbf{k}
\end{aligned}
$$

such that a fault in the $l$-th component $m_{l}(t) \neq 0$ can only affect the residual $r_{l}(t)$ and no other residual $r_{j}(t)(j \neq l)$ and

$$
\left\|r_{l}\right\|_{2, E}=\left\{\mathbb{E}\left[\int_{0}^{\infty} r_{l}^{\prime}(t) r_{l}(t) d t \mid\left(x_{0}, \alpha\right)\right]\right\}^{1 / 2}<\gamma\|d\|_{2}
$$

for all $d(t) \in \mathscr{L}_{2}$, and $\Pi \in \mathscr{D}_{\Pi}$, where $\gamma>0$ is a prescribed level of disturbance attenuation.

Below we first present a preliminary result on disturbance attenuation of the MJLS's.

Lemma 6.1 ( [7]): Let $\gamma$ be a given positive constant. If there exists a set of symmetric and positive-definite matrices $\mathfrak{R}=\left(R_{1}, \ldots, R_{N}\right)>0$ such that the following set of coupled LMIs hold for every $i \in \mathbf{N}$, namely

$$
\left[\begin{array}{cc}
J_{i} & C_{i}^{\prime} D_{d i}+R_{i} B_{d i} \\
* & D_{d i}^{\prime} D_{d i}-\gamma^{2} I
\end{array}\right]<0
$$

where $J_{i}=A_{i}^{\prime} R_{i}+R_{i} A_{i}+\sum_{j=1}^{N} \pi_{i j} R_{j}+C_{i}^{\prime} C_{i}$, then the system (17) with $u(t)=0$ and $m_{j}(t)=0, j \in \mathbf{k}$ is stochastically stable and for zero initial conditions satisfies the inequality $\|y\|_{2, E}<\gamma\|d\|_{2}$.

A system that satisfies the above conditions is said to be stochastically stable with a $\gamma$-disturbance attenuation property. In the next lemma, we consider the effects of uncertainties on the mode transition matrix $\Pi$ for analyzing the stochastic stability properties of system (17).

Lemma 6.2: Let $\gamma$ be a given positive constant. Let there exists a set of symmetric and positive-definite matrices $\mathfrak{R}=$ $\left(R_{1}, \ldots, R_{N}\right)>0$ and $\left\{\xi_{i j}>0, i, j \in \mathbf{N}, i \neq j\right\}$ such that the set of coupled LMIs holds for every $i \in \mathbf{N}$, that is

$$
\left[\begin{array}{ccc}
Q_{i} & C_{i}^{\prime} D_{d i}+R_{i} B_{d i} & M_{i} \\
* & D_{d i}^{\prime} D_{d i}-\gamma^{2} I & 0 \\
* & * & -\Xi_{i}
\end{array}\right]<0
$$


where $Q_{i}=A_{i}^{\prime} R_{i}+R_{i} A_{i}+\sum_{j=1}^{N} \bar{\pi}_{i j} R_{j}+C_{i}^{\prime} C_{i}+$ $\sum_{j=1, j \neq i}^{N} \frac{\xi_{i j}}{4} \epsilon_{i j}^{2} I, \quad M_{i}=\left[R_{i}-R_{1}, \cdots, R_{i}-\right.$ $\left.R_{i-1}, R_{i}-R_{i+1}, \cdots, R_{i}-R_{N}\right], \quad$ and $\Xi_{i}=$ $\operatorname{diag}\left(\xi_{i 1} I, \ldots, \xi_{i(i-1)} I, \xi_{i(i+1)} I, \ldots, \xi_{i N} I\right)$. It now follows that the uncertain system (17) with $u(t)=0$ and $m_{j}(t)=0, j \in \mathbf{k}$ is stochastically stable. Moreover, for zero initial conditions the system satisfies the inequality $\|y\|_{2, E}<\gamma\|d\|_{2}$ for all $\Pi \in \mathscr{D}_{\Pi}$.

Proof: According to Lemma 6.1, the uncertain system (17) with $u(t)=0$ and $m_{j}(t)=0, j \in \mathbf{k}$ is stochastically stable with a $\gamma$-disturbance attenuation property if

$$
\begin{aligned}
& A_{i}^{\prime} R_{i}+R_{i} A_{i}+\sum_{j=1}^{N}\left(\bar{\pi}_{i j}+\Delta \pi_{i j}\right) R_{j}+C_{i}^{\prime} C_{i}- \\
& \left(C_{i}^{\prime} D_{d i}+R_{i} B_{d i}\right)\left(D_{d i}^{\prime} D_{d i}-\gamma^{2} I\right)^{-1}\left(C_{i}^{\prime} D_{d i}+R_{i} B_{d i}\right)^{\prime}<0
\end{aligned}
$$

for all $i \in \mathbf{N}$. The above inequality can be rewritten as [33]

$$
\begin{aligned}
& A_{i}^{\prime} R_{i}+R_{i} A_{i}+\sum_{j=1}^{N} \bar{\pi}_{i j} R_{j}+C_{i}^{\prime} C_{i}- \\
& \left(C_{i}^{\prime} D_{d i}+R_{i} B_{d i}\right)\left(D_{d i}^{\prime} D_{d i}-\gamma^{2} I\right)^{-1}\left(C_{i}^{\prime} D_{d i}+R_{i} B_{d i}\right)^{\prime} \\
& +\sum_{j=1, j \neq i}^{N}\left[\frac{1}{2} \Delta \pi_{i j}\left(R_{j}-R_{i}\right)+\frac{1}{2} \Delta \pi_{i j}\left(R_{j}-R_{i}\right)\right]<0
\end{aligned}
$$

The above inequality holds for all $\left|\Delta \pi_{i j}\right| \leq \epsilon_{i j}$ if there exist $\xi_{i j}>0, i, j \in \mathbf{N}, i \neq j$ such that

$$
\begin{aligned}
& A_{i}^{\prime} R_{i}+R_{i} A_{i}+\sum_{j=1}^{N} \bar{\pi}_{i j} R_{j}+C_{i}^{\prime} C_{i} \\
& -\left(C_{i}^{\prime} D_{d i}+R_{i} B_{d i}\right)\left(D_{d i}^{\prime} D_{d i}-\gamma^{2} I\right)^{-1}\left(C_{i}^{\prime} D_{d i}+R_{i} B_{d i}\right)^{\prime} \\
& +\sum_{j=1, j \neq i}^{N}\left[\frac{\xi_{i j}}{4} \epsilon_{i j}^{2} I+\frac{1}{\xi_{i j}}\left(R_{j}-R_{i}\right)^{2}\right]<0
\end{aligned}
$$

It can be shown easily that the above is equivalent to the inequality (22) by using the Schur complement. This completes the proof of this lemma.

We are now in the position to derive our sufficient conditions for determining the solvability of the HEFPRG for an uncertain MJLS.

Theorem 6.3: The HEFPRG has a solution for the MJLS (17) with an uncertain mode transition matrix according to (18) if there exist $k$ outer MS-detectable unobservability subspaces $\mathcal{S}_{1}^{j^{*}}=\inf \mathfrak{S}_{1}\left(\mathfrak{A}, \mathfrak{C}, \sum_{v=1}^{N} \sum_{l=1, l \neq j}^{k} \mathcal{L}_{v}^{l}\right), j \in \mathbf{k}$ such that

$$
\mathcal{S}_{1}^{j^{*}} \bigcap \mathcal{L}_{i}^{j}=0, \quad i \in \mathbf{N}, j \in \mathbf{k}
$$

as well as the matrices $T_{i j}$, positive-definite matrices $R_{i j}, i \in$ $\mathbf{N}, j \in \mathbf{k}$, and $\left\{\xi_{i l}^{j}>0, i, l \in \mathbf{N}, i \neq l, j \in \mathbf{k}\right\}$ such that

$$
\left[\begin{array}{ccc}
Q_{i j} & \Upsilon_{i j} & \Theta_{i j} \\
* & D_{d i}^{\prime} H_{i j}^{\prime} H_{i j} D_{d i}-\gamma^{2} I & 0 \\
* & * & -\Xi_{i j}
\end{array}\right]<0
$$

for all $i \in \mathbf{N}, j \in \mathbf{k}$ with $Q_{i j}=A_{i j}^{\prime} R_{i j}+M_{i j}^{\prime} T_{i j}^{\prime}+$ $R_{i j} A_{i j}+T_{i j} M_{i j}+\sum_{l=1}^{N} \bar{\pi}_{i l} R_{l j}+M_{i j}^{\prime} M_{i j}+\sum_{l=1, l \neq i}^{N} \frac{\xi_{i l}^{j}}{4} \epsilon_{i l}^{2} I$, $\Upsilon_{i j}=-M_{i j}^{\prime} H_{i j} D_{d i}-R_{i j} P_{j} D_{i j} D_{d i}-R_{i j} P_{j} B_{d i}-T_{i j} H_{i j} D_{d i}$ $\Theta_{i j}=\left[R_{i j}-R_{1 j}, \cdots, R_{i j}-R_{(i-1) j}, R_{i j}-R_{(i+1) j}, \cdots, R_{i j}-\right.$
$\left.R_{N j}\right], \Xi_{i j}=\operatorname{diag}\left(\xi_{i 1}^{j} I, \ldots, \xi_{i(i-1)}^{j} I, \xi_{i(i+1)}^{j} I, \ldots, \xi_{i N}^{j} I\right)$ and where $P_{j}$ is the canonical projection of $\mathcal{X}$ on $\mathcal{X} / \mathcal{S}_{1}^{j^{*}}$, the pairs $\left(M_{i j}, A_{i j}\right), i \in \mathbf{N}, j \in \mathbf{k}$ are the factor system of the pairs $\left(C_{i}, A_{i}\right), i \in \mathbf{N}$ on $\mathcal{X} / \mathcal{S}_{1}^{j^{*}}, H_{i j}$ is the solution to $\operatorname{Ker} H_{i j} C_{i}=\mathcal{S}_{1}^{j^{*}}+\operatorname{Ker} C_{i}$ and $\mathcal{S}_{1}^{j^{*}}=<<\bigcap_{l=1}^{N} \operatorname{Ker} H_{l j} C_{l} \mid A_{i}+$ $D_{i j} C_{i}>>_{i \in \mathbf{N}}, j \in \mathbf{k}$.

Proof: Given $P_{j}$ as the canonical projection of $\mathcal{X}$ on $\mathcal{X} / \mathcal{S}_{1}^{j^{*}}$, let $M_{i j}, i \in \mathbf{N}$ be a unique solution to $M_{i j} P_{j}=H_{i j} C_{i}$ and define $A_{i j}=\left(A_{i}+D_{i j} C_{i}: \mathcal{X} / \mathcal{S}_{1}^{j^{*}}\right), i \in \mathbf{N}$. Let $G_{i j}=R_{i j}^{-1} T_{i j}, i \in \mathbf{N}, j \in \mathbf{k}$ where $T_{i j}$ and $R_{i j}$ are the solutions to the inequality (24). Define $F_{i j}=A_{i j}+G_{i j} M_{i j}$, $E_{i j}=P_{j}\left(D_{i j}+P_{j}^{-r} G_{i j} H_{i j}\right)$ and $K_{i j}=P_{j} B_{i}$ for $i \in \mathbf{N}, j \in$ k. Furthermore, define $e_{j}(t)=w_{j}(t)-P_{j} x(t)$, so that by using (19) we have

$$
\begin{aligned}
\dot{e}_{j}(t)= & F_{i j} w_{j}(t)-E_{i j} y(t)+K_{i j} u(t) \\
& -P_{j}\left(A_{i} x(k)+B_{i} u(k)+\sum_{l=1}^{k} L_{i}^{l} m_{l}(t)+B_{d i} d(t)\right) \\
= & \left(A_{i j}+G_{i j} M_{i j}\right) e_{j}(t)-P_{j} L_{i}^{j} m_{j}(t) \\
& -P_{j} D_{i j} D_{d i} d(t)-P_{j} B_{d i} d(t)-G_{i j} H_{i j} D_{d i} d(t)
\end{aligned}
$$

Furthermore, we have $r_{j}(t)=M_{i j} w_{j}(t)-H_{i j} y(t)=$ $M_{i} e_{j}(t)-H_{i j} D_{d i} d(t)$. Therefore, the residual $r_{j}$ is only affected by the fault $m_{j}$ and according to Lemma 6.2 and inequality (24), the inequality (20) holds for all the residuals $r_{j}(t), j \in \mathbf{k}$.

Remark 8: It should be noted that the above theorem results can be easily extended to the case where $\lambda(t)$ is not irreducible. In that case, similar filters as in (9) with timevarying dimensions are required and for each initial mode $i_{0} \in \Phi_{\alpha}$ the solvability conditions of the HEFPRG depend on the $\left|\mathrm{N}_{\alpha}\right|+1$ unobservability subspaces for classes $\mathrm{N}_{\alpha} \cup \alpha$.

Once the residual signals $r_{j}(t), j \in \mathbf{k}$ are constructed and generated, the final step in developing a reliable fault detection and isolation strategy deals with the residual evaluation process which involves determining the evaluation functions $J_{r_{j}}$ and their associated thresholds $J_{t h_{j}}$. Once the thresholds and evaluation functions are selected, the occurrence of a fault can be detected and isolated by using the following decision logics, namely $J_{r_{j}}>J_{t h_{j}} \Longrightarrow m_{j} \neq 0, \quad j \in \mathbf{k}$.

Finally, it should be emphasized that the fault sensitivity of the residuals can be improved by explicitly incorporating fault sensitivity in the design process. However, this topic is beyond the scope of the present work and is left as a topic of future research.

\section{CONCLUSIONS}

A geometric approach to the problem of fault detection and isolation (FDI) of continuous-time linear Markovian jump systems with (MJLSD's) and without time-delays (MJLS's) is developed in this paper. Starting with a new geometric characterization of the unobservable subspace of a Markovian jump system, the concept of unobservability subspaces is formalized and an algorithm for constructing these subspaces is presented. By invoking the notion of an unobservability subspace, the necessary and sufficient conditions for solving 
the fundamental problem of residual generation (FPRG) for Markovian jump systems is formally derived and investigated. Moreover, for MJLSD's, sufficient conditions for solvability of the FPRG are derived by utilizing only finite dimensional tools. For uncertain MJLS's, an $H_{\infty}$-based FDI strategy is proposed and developed where a set of residual signals are constructed such that each residual is only affected by one fault and is decoupled from the others while the $H_{\infty}$ norm of the transfer function between the unknown input (additive external disturbance in the input and output channels) and the residual signals is guaranteed to be less than a prescribed desired value. One of the future directions of research will be in investigating fault sensitivity for the MJLS's and the MJLSD's.

\section{ACKNOWLEDGEMENTS}

The authors would like to express their appreciations and sincere thanks to the reviewers (and in particular to Reviewer \#2) for their constructive and useful comments and suggestions that have significantly improved the presentation of the results in this work.

\section{REFERENCES}

[1] R. J. Patton, P. M. Frank, and R. N. Clark, Fault Diagnosis in Dynamic Systems, Theory and Applications. New York: Control Engineering series, Prentice-Hall, 1989.

[2] M. A. Massoumnia, G. C. Verghese, and A. S. Willsky, "Failure detection and identification," IEEE Trans. Autom. Control, vol. 34, no. 3, pp. 316-321, 1989.

[3] J. Gertler and D. Singer, "A new structural framework for parity equation based failure detection and isolation," Automatica, vol. 26, pp. 381-388, 1990.

[4] M. Basseville, "Detecting changes in signals and systems - a survey," Automatica, vol. 24, no. 3, pp. 309-326, 1988.

[5] J. Gertler, "Analytical redundacy methods in failure detection and isolation," Proceeding of IFAC Fault Detection, Supervision and Safety for Technical Processes, pp. 9-21, 1991.

[6] C. de Souza and M. Fragoso, " $\mathrm{H}_{\infty}$ filtering for Markovian jump linear systems," International Journal of Systen Science, vol. 33, pp. 909-915, 2002.

[7] E.-K. Boukas, Stochastic Switching Systems: Analysis and Design. Boston: Birkhauser, 2006.

[8] O. L. V. Costa, M. D. Fragoso, and R. P. Marques, Discrete-Time Markov Jump Systems. London: Springer-Verlag, 2005.

[9] E. F. Costa, J. B. R. do Val, and M. D. Fragoso, "A new approach to detectability of discrete-time infinite Markov jump linear systems," SIAM J. Control Optim., vol. 43, pp. 2132-2156, 2005.

[10] V. Gupta, R. M. Murray, and B. Hassibi, "On the control of jump linear Markov systems with Markov state estimation," Proceeding of American Control Conference, pp. 2893-2898, 2003.

[11] Z. Jin, V. Gupta, and R. M. Murray, "State estimation over packet dropping networks using multiple description coding," Automatica, vol. 42, no. 9, pp. 1441-1452, 2006.

[12] E. K. Boukas and Z. K. Liu, "Robust $\mathrm{H}_{\infty}$ control of discrete-time Markovian jump linear systems with mode-dependent time-delays," IEEE Trans. Autom. Control, vol. 46, no. 12, pp. 1918-1924, 2001.

[13] S. $\mathrm{Xu}$, T. Chen, and J. Lam, "Robust $\mathrm{H}_{\infty}$ filtering for uncertain Markovain jump systems with mode-dependent time delays," IEEE Trans. Autom. Control, vol. 48, no. 5, pp. 900-907, 2003.

[14] E.-K. Boukas, Deterministic and Stochastic Time Delay Systems. Boston: Birkhauser, 2002.

[15] P. Zhang, S. X. Ding, P. M. Frank, and M. Sader, "Fault detection of networked control systems with missing measurements," Proceeding of the $5^{\text {th }}$ Asian Control Conference, pp. 1258-1263, 2004.

[16] M. Zhong, H. Ye, P. Shi, and G. Wang, "Fault detection for Markovian jump systems," IEE Proc. Control Theory Application, vol. 152, no. 4 pp. 397-402, 2005.

[17] Z. Mao, B. Jiang, and P. Shi, " $\mathrm{H}_{\infty}$ fault detection filter design for networked control systems modeled by discrete Markovian jump systems," IET Control Theory and Application, vol. 1, no. 5, pp. 1336-1343, 2007.
[18] M. Zhong, J. Lam, S. X. Ding, and P. Shi, "Robust fault detection of Markovian jump systems," Circuits Systems Signal Processing, vol. 23, no. 5, pp. 387-407, 2008.

[19] H. Wang, C. Wang, H. Gao, and L. Wu, "An LMI approach to fault detection and isolation filter design for Markovian jump system with mode-dependent time-delays," Proceedings of the American Control Conference, pp. 5686-5691, 2006.

[20] N. Meskin and K. Khorasani, "Geometric approach to robust fault detection and isolation of Markovian jump systems," Proceeding of American Control Conference, pp. 2822-2827, 2008.

[21] _ "Fault detection and isolation of discrete-time Markovian jump linear systems with application to a network of multi-agent systems having imperfect communication channels," Automatica, vol. 45, no. 9, pp. 2032-2040, 2009.

[22] E. F. Costa and J. B. R. do Val, "On the observability and detectability of continuous-time Markov jump linear systems," SIAM J. Control Optim., vol. 41, no. 4, pp. 1295-1314, 2002.

[23] J. Bokor and G. Balas, "Detection filter design for LPV systems: A geometric approach," Automatica, vol. 40, no. 3, pp. 511-518, 2004.

[24] H. Hammouri, P. Kabore, and M. Kinnaert, "A geometric approach to fault detection and isolation for bilinear systems," IEEE Trans. Autom. Control, vol. 6, no. 9, pp. 1451-1455, 2001.

[25] N. Meskin and K. Khorasani, "Robust fault detection and isolation of time-delay systems using a geometric approach," Automatica, vol. 45, no. 6, pp. 1567-1573, 2009.

[26] _ "Fault detection and isolation of distributed time-delay systems,", IEEE Trans. Autom. Control, 2009, to appear.

[27] — "Unobservability subspaces for continuous-time Markovian jump systems with application to fault diagnosis," Proceeding of the 7th IFAC Symposium on Fault Detection, Supervision and Safety of Technical Processes, pp. 953-958, 2009.

[28] W. M. Wonham, Linear Multivariable Control - A Geometric Approach, 3rd ed. New York:Springer, 1985.

[29] G. Basile and G. Marro, Controlled and Conditioned Invariant Subspaces in Linear System Theory. Prentice-Hall, 1992.

[30] M. Kijima, Markov Processes for Stochastic Modeling. Chapman \& Hall, 1997.

[31] C. De Persis and A. Isidori, "A geometric approach to nonlinear fault detection and isolation," IEEE Trans. Autom. Control, vol. 46, no. 6, pp. 853-865, 2001

[32] G. Basile and G. Marro, "On the robust controlled invariant," Systems \& Control Letters, vol. 9, no. 3, pp. 191-195, 1987.

[33] J. Xiong, J. Lam., H. Gao, and D. W. C. Ho, "On robust stabilization of Markovian jump systems with uncertain switching probabilities," Automatica, vol. 41, no. 5, pp. 897-903, 2005.

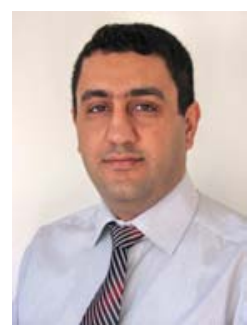

Nader Meskin received his B.Sc. from Sharif University of Technology, Tehran, Iran, in 1998, his M.Sc. from the University of Tehran, Tehran, Iran in 2001, and obtained his Ph.D. in Electrical and Computer Engineering in 2008 from Concordia University, Montreal, Canada. He is currently a postdoctoral fellow at Concordia University. Dr. Meskin research interests include Fault Detection and Isolation (FDI), autonomous network of unmanned vehicles, time delay systems, and Markovian jump systems.

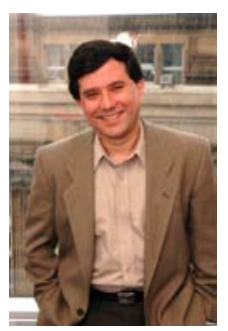

K. Khorasani (M'85) received the B.S., M.S., and Ph.D. degrees in Electrical and Computer Engineering from the University of Illinois at UrbanaChampaign in 1981, 1982 and 1985, respectively. From 1985 to 1988 he was an Assistant Professor at the University of Michigan at Dearborn and since 1988, he has been at Concordia University, Montreal, Canada, where he is currently a Professor and Concordia University Tier I Research Chair in the Department of Electrical and Computer Engineering. His main areas of research are in nonlinear and adaptive control, intelligent and autonomous control of networked unmanned systems, fault diagnosis, isolation and recovery (FDIR), neural network applications to pattern recognition, robotics and control, adaptive structure neural networks, and modeling and control of flexible link/joint manipulators. He has authored/co-authored over 300 publications in these areas. 